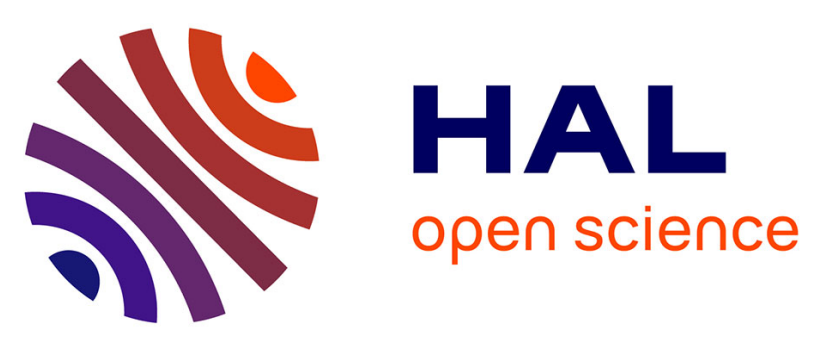

\title{
Meiotic Cells Counteract Programmed Retrotransposon Activation via RNA-Binding Translational Repressor Assemblies
}

Raphaelle Laureau, Annie Dyatel, Gizem Dursuk, Samantha Brown, Hannah Adeoye, Jia-Xing Yue, Matteo de Chiara, Anthony Harris, Elçin Ünal, Gianni Liti, et al.

\section{To cite this version:}

Raphaelle Laureau, Annie Dyatel, Gizem Dursuk, Samantha Brown, Hannah Adeoye, et al.. Meiotic Cells Counteract Programmed Retrotransposon Activation via RNA-Binding Translational Repressor Assemblies. Developmental Cell, 2021, 56, pp.22 - 35.e7. 10.1016/j.devcel.2020.11.008 . hal03455850

\section{HAL Id: hal-03455850 https://hal.science/hal-03455850}

Submitted on 29 Nov 2021

HAL is a multi-disciplinary open access archive for the deposit and dissemination of scientific research documents, whether they are published or not. The documents may come from teaching and research institutions in France or abroad, or from public or private research centers.
L'archive ouverte pluridisciplinaire HAL, est destinée au dépôt et à la diffusion de documents scientifiques de niveau recherche, publiés ou non, émanant des établissements d'enseignement et de recherche français ou étrangers, des laboratoires publics ou privés. 


\section{Developmental Cell}

\section{Meiotic Cells Counteract Programmed Retrotransposon Activation via RNA-Binding Translational Repressor Assemblies}

\section{Graphical Abstract}

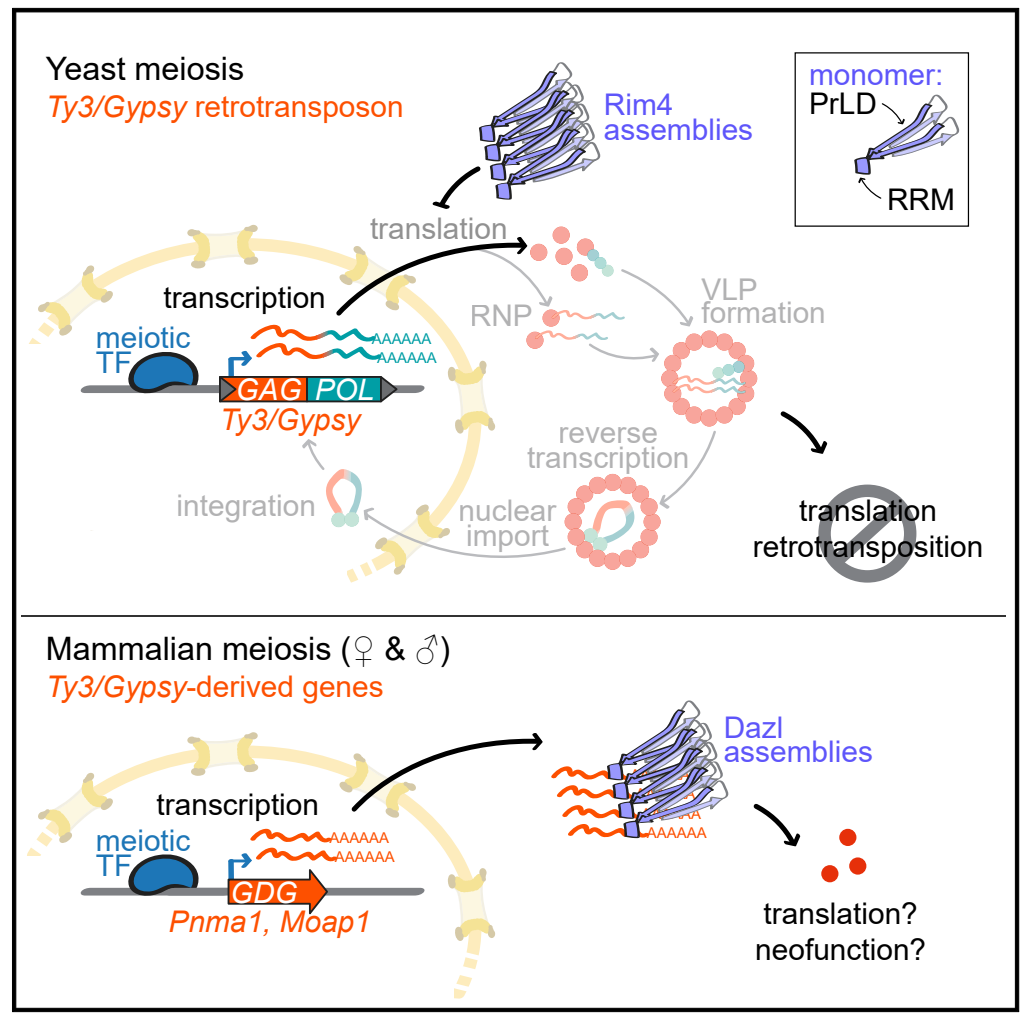

\section{Authors}

Raphaelle Laureau, Annie Dyatel, Gizem Dursuk, ..., Gianni Liti, lan R. Adams, Luke E. Berchowitz

\section{Correspondence}

leb2210@cumc.columbia.edu

\section{In Brief}

Retrotransposon proliferation poses a threat to germline integrity. Laureau et al., demonstrate that yeast Ty3/Gypsy LTRretrotransposons co-opt a host transcription factor during gametogenesis. In defense, developing gametes block retrotransposon translation. Mammalian genes derived from Tyз/Gypsy are regulated during meiosis via equivalent circuitry, suggesting that host-transposon conflicts could shape emerging gene function.

\section{Highlights}

- Ty3/Gypsy retrotransposons co-opt a transcription factor for its activation in meiosis

- Host amyloid-like translational repressors block the Туз/ Gypsy life cycle

- In mammalian gametogenesis, equivalent regulators govern Ty3/Gypsy-derived genes

- Host-transposon conflicts may shape the regulation of emerging genes 


\title{
Developmental Cell
}

Article

Meiotic Cells Counteract Programmed

Retrotransposon Activation via RNA-Binding Translational Repressor Assemblies

\author{
Raphaelle Laureau, ${ }^{1}$ Annie Dyatel, ${ }^{1}$ Gizem Dursuk, ${ }^{1}$ Samantha Brown, ${ }^{1}$ Hannah Adeoye, ${ }^{1}$ Jia-Xing Yue, ${ }^{2}$ \\ Matteo De Chiara, ${ }^{2}$ Anthony Harris, ${ }^{3}$ Elçin Ünal, ${ }^{3}$ Gianni Liti, ${ }^{2}$ lan R. Adams, ${ }^{4}$ and Luke E. Berchowitz ${ }^{1,5, *}$ \\ ${ }^{1}$ Department of Genetics and Development, Hammer Health Sciences Center, Columbia University Irving Medical Center, New York, NY \\ 10032, USA \\ 2Université Côte d'Azur, CNRS, INSERM, IRCAN, Nice 06107, France \\ 3Department of Molecular and Cell Biology, University of California Berkeley, Berkeley, CA 94720, USA \\ ${ }^{4}$ MRC Human Genetics Unit, MRC Institute of Genetics and Molecular Medicine, University of Edinburgh, Edinburgh EH4 2XU, UK \\ ${ }^{5}$ Lead Contact \\ *Correspondence: leb2210@cumc.columbia.edu \\ https://doi.org/10.1016/j.devcel.2020.11.008
}

\section{SUMMARY}

Retrotransposon proliferation poses a threat to germline integrity. While retrotransposons must be activated in developing germ cells in order to survive and propagate, how they are selectively activated in the context of meiosis is unclear. We demonstrate that the transcriptional activation of Ty3/Gypsy retrotransposons and host defense are controlled by master meiotic regulators. We show that budding yeast Ty3/Gypsy co-opts binding sites of the essential meiotic transcription factor Ndt80 upstream of the integration site, thereby tightly linking its transcriptional activation to meiotic progression. We also elucidate how yeast cells thwart Ty3/Gypsy proliferation by blocking translation of the retrotransposon mRNA using amyloid-like assemblies of the RNA-binding protein Rim4. In mammals, several inactive Ty3/Gypsy elements are undergoing domestication. We show that mammals utilize equivalent master meiotic regulators (Stra8, Mybl1, Dazl) to regulate Ty3/Gypsy-derived genes in developing gametes. Our findings inform how genes that are evolving from retrotransposons can build upon existing regulatory networks during domestication.

\section{INTRODUCTION}

The relationship between organisms and transposable elements is complex and fluctuating. For the organism, transposable elements can act as a reservoir for novel genes and drive genomic plasticity, but they are also key drivers of genome instability. Insertions can rearrange regulatory networks and alter gene expression patterns (Chuong et al., 2017; Mita and Boeke, 2016; Rebollo et al., 2012). More dangerously, insertion of transposable elements can disrupt genetic information, give rise to heterologies, and potentially provide sites for ectopic recombination (Warren et al., 2015). For the element, if it is to survive and proliferate, it must do so with minimal detriment and possible benefit to the host (Cosby et al., 2019; Haig, 2016).

Retrotransposons are a class of transposable elements that propagate through an RNA intermediate via a copy-and-paste mechanism. This mechanism preserves the original element while generating DNA copies, which are free to invade other loci. Retrotransposon proliferation has seemingly no intrinsic limit and poses an acute burden to the host genome. This burden is reflected in the repetitive fraction of genomes, which is primarily composed of retrotransposon-derived sequences. In this study, we focus on understanding the biology underpinning pro- liferation of a class of long terminal repeats retrotransposons (LTR-retrotransposons), Ty3/Gypsy, during meiosis.

LTR-retrotransposons are divided into four superfamilies, based on structure and phylogeny: Ty1/Copia, Ty3/Gypsy (both represented in all eukaryotic lineages), Bel-Pao (found in metazoans but not mammals), and endogenous retroviruses (ERVs), which are found only in vertebrates) (Wicker et al., 2007). Ty3/Gypsy and ERVs are closely related because many vertebrate retroviruses likely arose from Ty3/Gypsy retrotransposons that acquired the envelope gene from other viruses (Hayward, 2017). Although no active Ty3/Gypsy retrotransposons are found in mammalian genomes, several sequences derived from Ty3/Gypsy are present, many of which have been domesticated for cellular functions (Volff, 2009).

The Ty3/Gypsy life cycle is much like that of a retrovirus except that it occurs entirely within the cell. It is transcribed into an mRNA that encodes 2 polyproteins: Gag3 and Gag3-Pol3 (Figure $1 \mathrm{~A})$. Gag3 is the precursor to the capsid (CA) and nucleocapsid (NC) proteins and $\mathrm{Pol} 3$ is the precursor to a protease (PR), a reverse transcriptase- $\mathrm{RNaseH}(\mathrm{RT}-\mathrm{RH})$, and an integrase (IN). In most cases, only Gag3 polyprotein is translated, while Gag3-Pol3 is only produced by a frameshift that occurs in 5\%$10 \%$ of translation events (Farabaugh et al., 1993) (Figure 1A). 
A
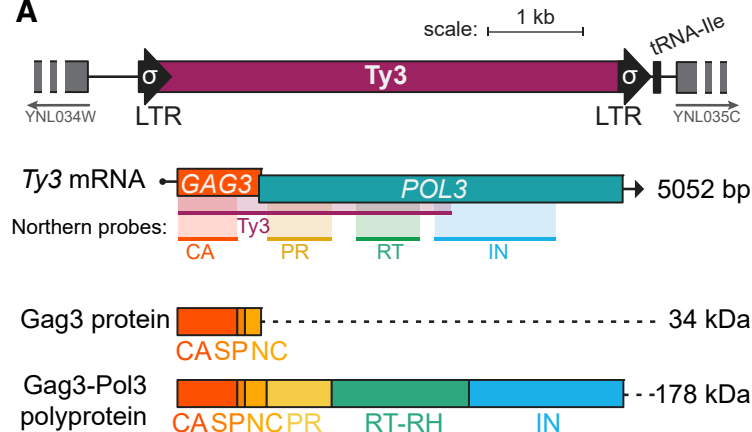

C
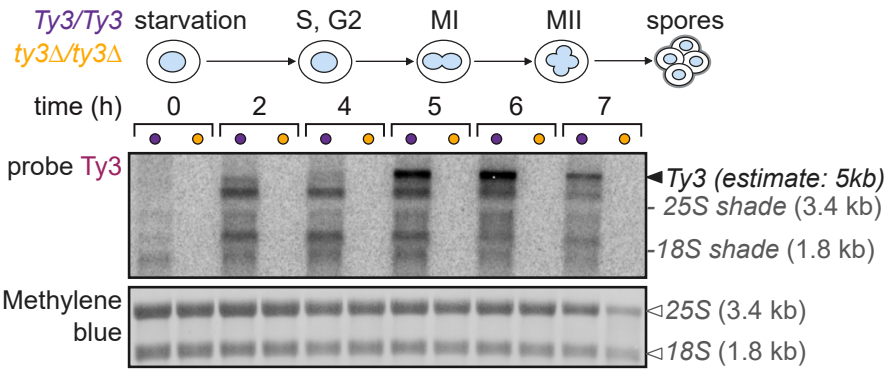

E

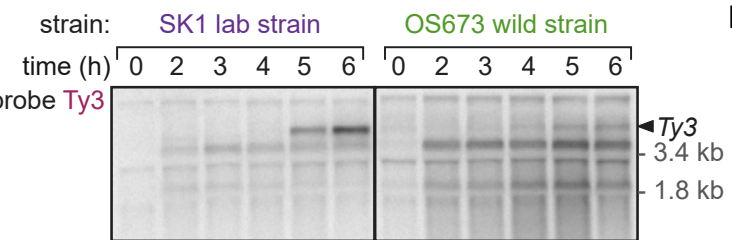

Methylene

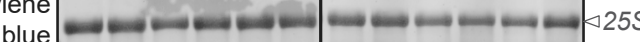

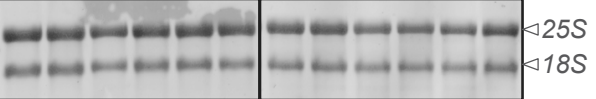

B

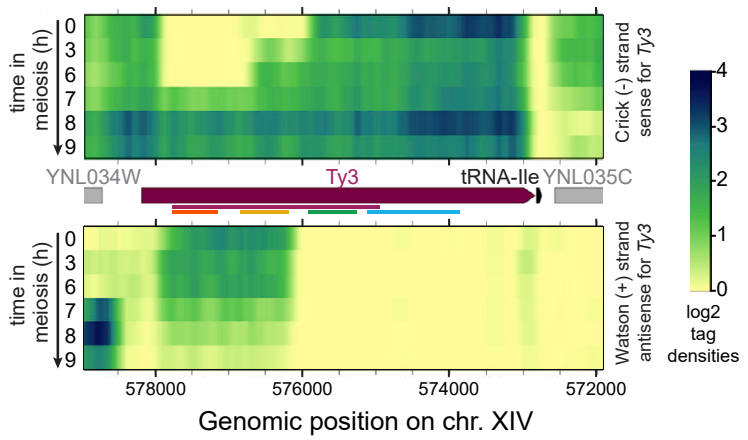

D

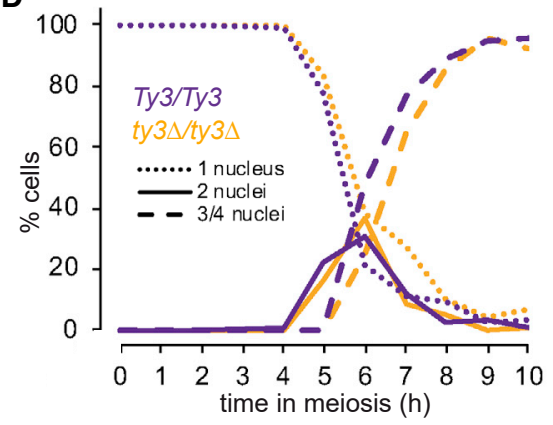

G

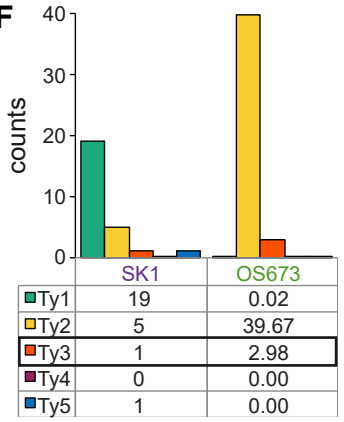

Figure 1. Ty3 Expression Is Coordinated with Meiotic Progression

(A) Diagram of the SK1 Ty3 element DNA locus, full-length mRNA, and two polyproteins, Gag3 (precursor of CA-capsid SP-spacer and NC-nucleocapsid proteins) and Gag3-Pol3 (precursor of the aforementioned proteins and of PR-protease, RT-RH-reverse transcriptase-RNaseH and IN-integrase proteins). Colored lines indicate the positions of the Northern probes used in this study: Ty3 probe (maroon), CA probe (orange), PR probe (yellow), RT probe (green), IN probe (blue). (B) $\log _{2}$ tag density of polyA-RNA-seq reads of Ty3 locus from synchronized meiotic cells (Crick strand top). Cells harboring NDT80-IN (NDT80 inducible strain $\mathrm{B} 119)$ were sporulated at $30^{\circ} \mathrm{C}$. Shown are the time points at $0,3,6,7,8$, and $9 \mathrm{~h}$ post transfer to sporulation medium. At $6 \mathrm{~h}$ when cells had arrested in $\mathrm{G} 2 \mathrm{due}$ to the lack of Ndt80 meiotic transcription factor, they were released from the block by addition of $1 \mu \mathrm{M} \beta$-estradiol (biological replicates $=2$ ).

(C and D) Full-length Ty3 mRNA is expressed in meiosis. Sporulation was induced in wild type diploid strain (B47, purple) and in ty3 $\Delta$ diploid strain (B837, gold). (C) Ty3 mRNA and rRNA (loading control) levels are shown (S, premeiotic DNA replication; G2, meiotic prophase; MI, first meiotic division; MII, second meiotic division). Full-length Ty3 mRNA ( $5 \mathrm{~kb}$ ) is indicated by a black arrow. Empty arrowheads indicate $18 \mathrm{~S}$ and $25 \mathrm{~S} r R N A(1.8 \mathrm{and} 3.4 \mathrm{~kb})$, which are both reported on the probed blot and serve as size markers. (D) Kinetics of meiotic progression was determined via DAPI nuclear staining. Shown are representative results of $\geq 5$ biological replicates.

(E-G) Ty3 is activated during meiosis in wild S. cerevisiae. Wild type SK1 (B47, purple) and wild European strain OS673 (green, described in Peter et al., 2018) were induced to sporulate as in (C). (E) Ty3 mRNA and rRNA levels are shown. (F) The number of Ty elements in SK1 and OS673 are shown. (G) Kinetics of meiotic progression determined by DAPI nuclear staining (biological replicates $=2$ ).

Gag3 assembles with a Ty3 mRNA dimer and Gag3-Pol3 into a remarkable structure within the cell-the virus-like particle (VLP)-inside which the precursor proproteins mature into their final products and the Ty3 mRNA is reverse transcribed into cDNA (Garfinkel et al., 1985). This cDNA copy is bound to integrase proteins and can enter the nucleus via the nuclear pore to integrate in the genome. Ty3 integration events require binding to polymerase III, and thus, frequently occur in close proximity to tRNA genes (Qi et al., 2012).

For a retrotransposon to increase its copy numbers and proliferate through generations, it must to do so in cells that will ultimately become gametes. For this reason, retrotransposons tend to be expressed in the developing germline, including during meiosis (Crichton et al., 2014; Ribeiro-dos-Santos et al., 


\section{Developmental Cell}

1997). However, as retrotransposon mobilization in these cells can cause heritable mutations and trans-generational genetic instability, organisms have evolved elegant multilayered mechanisms, which act at various stages of the retrotransposon cycle to protect their germlines (Crichton et al., 2014).

For example, transcription of retrotransposons can be repressed by DNA methylation, chromatin modifications, and transcriptional interference (for review (Crichton et al., 2014; Yang and Wang, 2016)). Post-transcriptional pathways, such as miRNA, siRNA, and piRNA, target retrotransposon mRNA for degradation (Bao and Yan, 2012; Dechaud et al., 2019; Yang and Wang, 2016) and RNA modification enzymes, such as cytidine deaminases, prevent reverse transcription of the retrotransposon mRNA into cDNA (Orecchini et al., 2018). Posttranslational regulatory mechanisms act in germ cells to target retrotransposon proteins for premature degradation (MacLennan et al., 2017). Beyond the obvious long-term consequences of uncontrolled retrotransposon proliferation, dysregulation of retrotransposon control can cause acute failures in gamete development. Disruption of defense mechanisms in mice or Drosophila leads to sterility (Barau et al., 2016; Bourc'his and Bestor, 2004; Wang et al., 2018), and hyperactivation of retrotransposons causes fetal oocyte attrition in mice (Malki et al., 2014).

Here, we show that Ty3/Gypsy elements can exploit the genomic environment at their integration sites to ensure transcriptional activation during meiotic divisions. Our results illustrate a mechanism by which retrotransposons can restrict their expression to a developmental context in which activation is beneficial. We also identify an opposing mechanism by which cells defend their genome against meiotic retrotransposition. Developing budding yeast Saccharomyces cerevisiae gametes utilize amyloid-like RNA-binding protein assemblies to repress Ty3 translation, thereby halting the proliferation cycle. We find that both the co-opting of meiotic regulators by Ty3/Gypsy elements and their interaction with assembly-forming RNA-binding proteins are strategies that are also employed by Gypsy-derived genes in mammals. Our findings highlight the likely hundreds of millions of years of perpetual interactions among retrotransposons, host RNA-binding proteins, and genomes.

\section{RESULTS}

\section{Ty3 Is Expressed in Coordination with Meiotic Progression}

We first determined whether retrotransposons are transcriptionally active during $S$. cerevisiae meiosis (strains used in this study are described in Table S1). We performed RNA-seq at hourly time points from cells undergoing synchronous meiosis using the NDT80 block-release system (Benjamin et al., 2003; Carlile and Amon, 2008). There was only one full-length copy of Ty3 in the SK1 strain, and we observed that this locus was transcribed in the sense orientation, specifically during the meiotic divisions (Figures $1 \mathrm{~A}$ and 1B). We also observed an antisense RNA at the onset of meiosis that declined to almost undetectable levels as meiosis proceeded. These data led us to hypothesize that transcription of the full-length Ty3 is repressed in mitotic cells but is activated during the meiotic divisions.

Only a full-length retrotransposon mRNA can complete the steps necessary for proliferation. While our RNA-seq data imply that the Ty3 locus is transcribed in meiosis, we sought to differentiate whether this was indicative of bona fide full-length transcripts or of cryptic inactive transcripts that span the locus. Using Northern blot analysis, we observed several Ty3 RNA isoforms (Figure 1C), consistent in size with the various isoforms spanning the locus in the RNA-seq (Figure 1B), including a prominent band of the size of a full-length transcript $(5 \mathrm{~kb})$. All of the RNA isoforms we observed were specific to Ty3 because none of them were produced in cells lacking Ty3 (ty3 3 ) (Figures 1C, $\mathrm{S} 1 \mathrm{~A}$, and $\mathrm{S} 1 \mathrm{~B})$. Using probe tiling, we were able to identify this top band as a full-length mRNA, containing both GAG3 and POL3 coding sequences (Figure S1C) and thus the potential capacity to propagate. Strikingly, full-length Ty 3 mRNA was expressed and reached maximal levels at the first meiotic division (Figures 1C and 1D).

We next determined whether meiotic transcriptional activation of Ty3 occurs in other S. cerevisiae isolates. Because rapid, efficient, and synchronous meiosis is a feature that is not shared by most lab strains, we decided to investigate a collection of wild diploid isolates (Peter et al., 2018) for sporulation efficiency and Ty3 activation (Table S2). These strains harbor varying Ty3 copy numbers. We found that full-length Ty3 transcription switches on at the time of meiotic divisions in at least one isolate, OS673, which harbors two full-length copies of Ty3 (Figures 1E1G; Table S2). This strain was isolated from a beetle in Hungary (Peter et al., 2018) and is of a completely different genetic background than SK1 ( 51,400 SNPS), allowing us to rule out the possibility that $T y 3$ activation in meiosis is a peculiarity that appeared during lab domestication. From these data, we conclude that Ty3 retrotransposons have evolved to activate, and potentially proliferate, during meiosis.

\section{Ty3 Expression Is Controlled by a Critical Meiotic Transcription Factor}

How is Ty3 transcription activated specifically at the onset of meiosis I? The transition from meiotic prophase to the first meiotic division is governed by the meiosis-specific transcription factor Ndt80 (Xu et al., 1995). Accordingly, we asked whether Ty3 is under the control of Ndt80. If Ty3 is a Ndt80 target, it would not be transcribed in meiotic cells deprived of Ndt80. Furthermore, Ty3 transcription should be selectively activated upon ectopic Ndt80 activity. We found that cells in which NDT80 is not induced-and are thus arrested in meiotic prophase-do not express Ty3 mRNA (Figures S2A and S2B). Additionally, in meiotic cells where NDT80 expression is delayed, Ty3 transcription exhibits a corresponding delay. We observed that Ty3 fulllength transcripts accumulate from 15 to 30 min before metaphase I and persist until exit from anaphase II (Figures 2A and 2B). These results imply that Ty3 is a target of Ndt80.

In further support of this idea, Ty3 is integrated downstream of two Ndt80 binding sites (79 bp and 269 bp) (Figure 2C). To investigate whether Ty 3 meiotic transcription activation is governed by its environment of integration rather than elements within its $5^{\prime}$ LTR, we asked if Ty3 is still transcribed in cells harboring a promoter-gene cassette (HIS3MX) between the $5^{\prime}$ LTR and the first proximal Ndt80 binding site (Figure S3A). We found that Ty3 was no longer transcribed when we separated it from its upstream genetic context (Figure S3B). To test whether Ndt80 directly activates Ty3, we mutated the two Ndt80 binding sites upstream of 
A

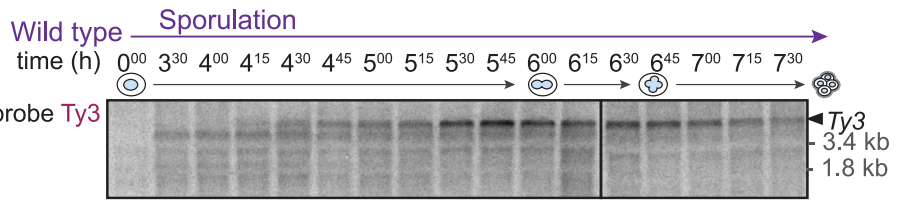

Methylene

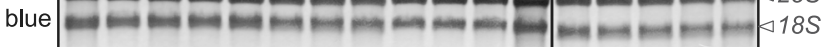

NDT80-IN_G2 arrest Release

time (h) $0^{00} 3^{30} 4^{00} 5^{00} 6^{00} 6^{15} 6^{30} 6^{45} 7^{00} 7^{15} 7^{30} 7^{45} 8^{00} 8^{15} 8^{30} 8^{45} 9^{00}$

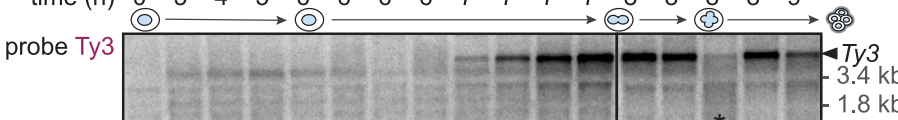

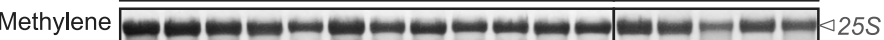

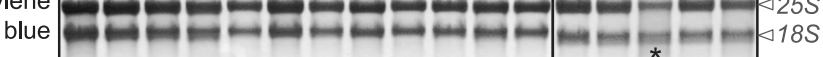

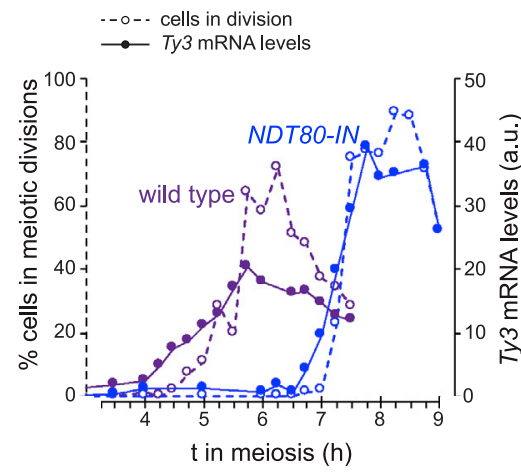

C

Ndt80 binding sites (CACAAA):

$\longrightarrow$-LTRo Ty3

mutated binding sites (AAAAAA):

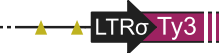

scale: $\longdiv { 5 0 0 \mathrm { bp } }$
D

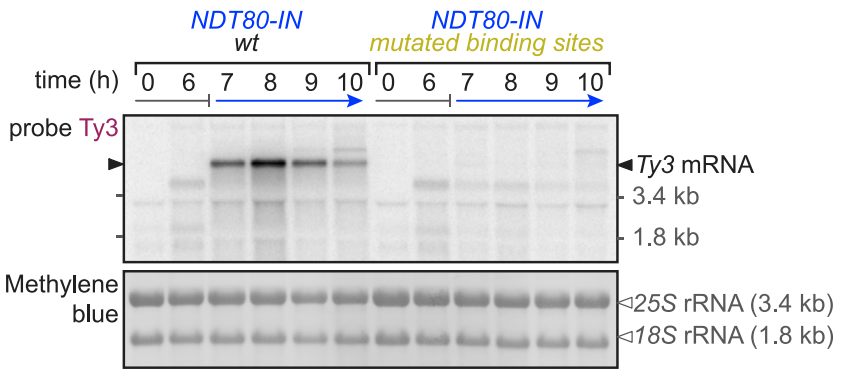

E

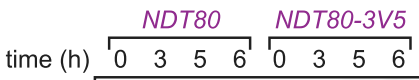

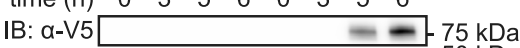

IB: $\alpha-P g k 1-1---150 \mathrm{kDa}$

$\mathbf{F}$

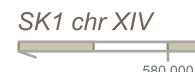

580,000

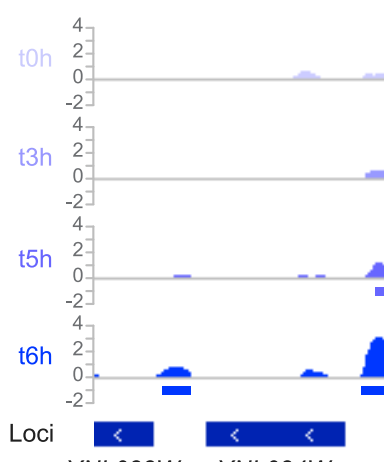

YNL033W

YNL034W
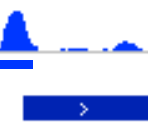

575,000
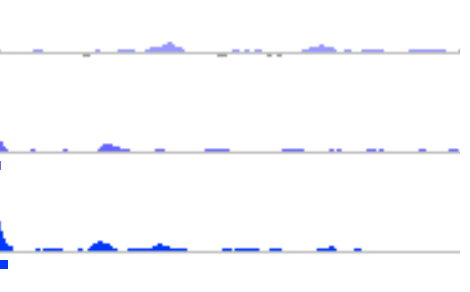

$T Y 3$

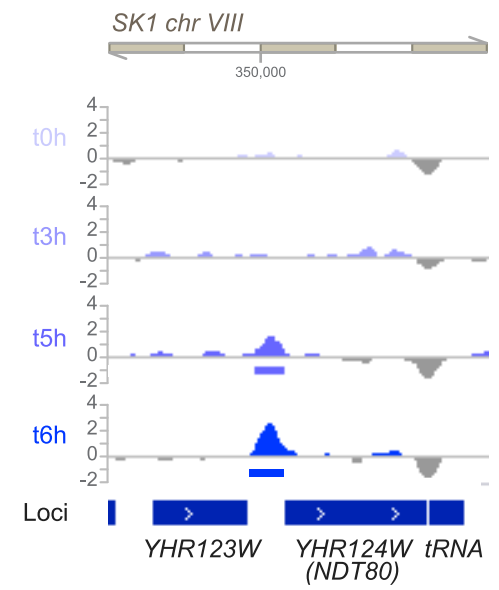

Figure 2. Ty3 Exploits Binding Sites of a Meiotic Transcription Factor from the Integration Environment (A and B)Ty3 expression is controlled by Ndt80

(A) Sporulation was induced in parallel in wild type (B47, purple), and in NDT80-IN (B48, blue, released from the G2 block at 6 h). Ty3 mRNA and rRNA levels are shown. Asterisk indicates a degraded RNA sample, which is excluded from the quantification shown in (B).

(B) Solid lines indicate quantification of RNA levels from (A), dashed lines indicate kinetics of meiotic divisions analyzed by tubulin immunofluorescence (quantification of cells containing meiotic spindles). Shown are representative results of $\geq 5$ biological replicates.

(C and D) Ty 3 transcription is directly activated from Ndt80 binding sites upstream of the integration site. Diagram of endogenous and mutated Ty 3 upstream context. (C) Blue triangles indicate Ndt80 binding sites (CACAAA) and yellow triangles indicated mutated binding sites (AAAAA) ( -79 and -269 bp upstream of Ty3). (D) Sporulation was induced in NDT80-IN strains containing the wild-type Ty3 Ndt80 binding sites (B1864, blue), or with mutated Ndt80 binding sites (B1885, yellow) as in (A) (in both strains, a kanMX marker was inserted 353 bp upstream the Ty3 $5^{\prime}$ LTR). Ty3 mRNA and $r$ RNA levels are shown. Shown are representative results of 2 biological replicates. 


\section{Developmental Cell}

Ty3 (CACAAA to AAAAAA) and assessed the effect of these mutations on meiotic Ty3 transcription (Figure 2C). We found that point mutation of these sequences specifically abolished fulllength meiotic Ty3 transcription (Figure 2D). To assess whether Ndt80 binds upstream of Ty3 during meiotic progression, we conducted a meiotic time course analysis of $\mathrm{Ndt} 80$ binding by ChIP-seq. We analyzed Ndt80 binding at 0, 3, 5, and $6 \mathrm{~h}$ in meiosis. We chose these time points because Ndt80 levels are highest at $\sim 5-6 \mathrm{~h}$ in meiosis (Figure 2E). In support of the hypothesis that Ty3 is transcriptionally activated by Ndt80, we found that Ndt80 binds upstream of Ty3 as meiosis progresses (Figure $2 \mathrm{~F}$ ). We conclude that Ty3 co-opts a host regulatory circuit to coordinate its activation with meiotic division and we predict that other retroelements could have evolved a similar strategy to acquire germline-specific activation.

The Ndt80 protein sequence as well as its binding site is conserved among yeasts from Saccharomyces to Candida (Nocedal et al., 2017). We thus sought to determine whether Ty3 control by Ndt80 is conserved among other Saccharomyces. We assessed the presence of Ndt80 binding sites within and upstream of full-length Ty3 elements in seven S. cerevisiae and five S. paradoxus genomes for which all Ty elements have been mapped and annotated (Yue et al., 2017) (spanning approximately 5 million years of evolution (Shen et al., 2018)) (Figure 3A). Among twenty full-length Ty3 elements, we found that thirteen harbor one or more $\mathrm{Ndt} 80$ binding sites within $1 \mathrm{~kb}$ upstream of their integration sites, including five cases where the $\mathrm{Ndt80}$ binding site is less than 250 bp upstream the integration site (Figure 3A). Within the 1-kb regions upstream of Ty3 elements, we observed that several of them harbor multiple Ndt80 binding sites. In total, we observed nineteen $\mathrm{Ndt} 80$ binding sites within the 20 -total kb, which is significantly higher than what would be expected by chance ( $Z$ score $2.7 ; p<0.01)$.

Because Ndt80 binding sites are abundant (about 8,000 per genome), we needed to ascertain whether the distance of a Ndt80 binding site from a locus is predictive of whether that locus is truly activated by Ndt80. Ndt80-responsive genes are well defined in SK1 meiosis (Chu et al., 1998). We first compared the enrichment of Ndt80 binding sites upstream of Ndt80 target genes and non-target genes and found a peak of enrichment within the 50 to 250-bp region upstream of the transcription start sites (TSS) in target genes (Figure 3B). We next asked whether the association between $\mathrm{Ty} 3$ elements and $\mathrm{Ndt} 80$ binding site resembles that of Ndt80 targets or non-targets. Because the small number of full-length Ty3 found in the Saccharomyces mentioned above gave us limited statistical power, we combined full-length elements with Ty3 solo-LTRs $(n=498)$, which are the vestiges of Ty3 integrations lost by homologous recombination between LTRs. We found that taken together, Ty3 elements showed a significant enrichment of Ndt80 binding sites 200 bp upstream of their integration site compared to non-targets $(p<$ 0.001 , Figure $3 \mathrm{~B}$ ). This result strongly supports the hypothesis that the co-opting of $\mathrm{Ndt80}$ by Ty3 elements is a recurring feature.

Ty3 Translation Is Repressed Specifically in Meiosis Ty3 has co-opted a meiotic control element to activate its transcription specifically during meiosis. We next sought to determine how yeast responds to protect its developing gametes from the potential threat of Ty 3 proliferation. Because the Ty 3 copy number seems to be relatively low and stable in yeast strains (Table S2), we reasoned that yeast cells might have evolved a defense mechanism that acts post-transcriptionally. We first wanted to ascertain whether Gag3 protein is produced in meiosis. We found that Gag3 was produced in our positive control-exposure of MATa SK1 haploid cells to $\alpha$-factor mating pheromone (Figure 4A) - a condition in which full-length Ty3 RNA was shown to be both transcribed and translated in another lab strain via the transcription factor Ste12 (Bilanchone et al., 1993). Hence, we confirmed that Ty3 is an active element in SK1. However, despite the presence of full-length Ty3 mRNA in meiosis, no detectable Gag3 protein was produced (Figure 4A). The absence of Gag3 protein in meiosis could be due to the lack of translation or rapid protein degradation. To test the latter possibility, we inhibited proteasomal function in meiotic cells. We found that either genetic (rpn6-1 (Isono et al., 2005)) or chemical inhibition (MG-132) of the proteasome did not result in Gag3 protein accumulation (Figure S4A). We concluded that the absence of Gag3 in meiosis is not due to degradation by the proteasome. Instead, our data support a model by which, in response to Ty3 transcription in meiosis, yeast cells respond by preventing translation of the mRNA thereby halting the retrotransposition cycle.

Because Ty3 mRNA can be translated upon exposure of MATa cells to $\alpha$-factor pheromone, we suspected that translational repression of Ty3 is specific to meiosis. To test this hypothesis, we ectopically expressed NDT80 in haploid mitotic cells to drive Ty3 transcription. In parallel, we depleted the mitotic repressor Sum1 that binds upstream of Ndt80 target genes to inhibit their inappropriate expression (Klutstein et al., 2010; Pierce et al., 2003; Winter, 2012). Selective depletion of Sum1 was achieved using the auxininducible degron system (Morawska and Ulrich, 2013). We found that when we expressed NDT80 in mitotic cells either in the presence or absence of Sum1 (Figure S4B), Ty3 was both transcribed and translated (Figure 4B). Ty3 mRNA and protein levels were much higher when Sum1 was depleted. We also observed that upon NDT80 activation in meiosis or mitosis, Ty3 mRNA levels were similar, while translation occurred only in the mitotic context. In addition, we observed that Ty3 mRNA dimers, which are indicative of VLP assembly (Cristofari et al., 1999; Feng et al., 2000), form upon NDT80 induction in mitosis but not in meiosis (Figure S4C). We conclude that the mechanism by which yeast represses translation of Ty 3 mRNA is meiosis specific. These data also provide additional support to the notion that this retrotransposon has co-opted the Sum1/Ndt80 regulon to restrict its expression to meiosis.

(E and F) Ndt80 binds directly upstream of Ty3 integration sites as meiosis progresses. Sporulation was induced in wild-type diploid strain (B47 no tag) and in NDT80-3V5 diploid strain (B1674). Shown are the time points 0, 3, 5, $6 \mathrm{~h}$ post-transfer to sporulation medium.

(E) Analysis of Ndt80 protein expression. Ndt80 (tagged with 3V5) and Pgk1 (loading control) protein levels are shown. Migration of molecular size standards is indicated on right as $\mathrm{kDa}$ values.

(F") Browser tracks showing Ndt80-3V5 ChIP-seq peaks (blue bars). Ty3 (left) and NDT80 (right, positive control) loci are shown. 


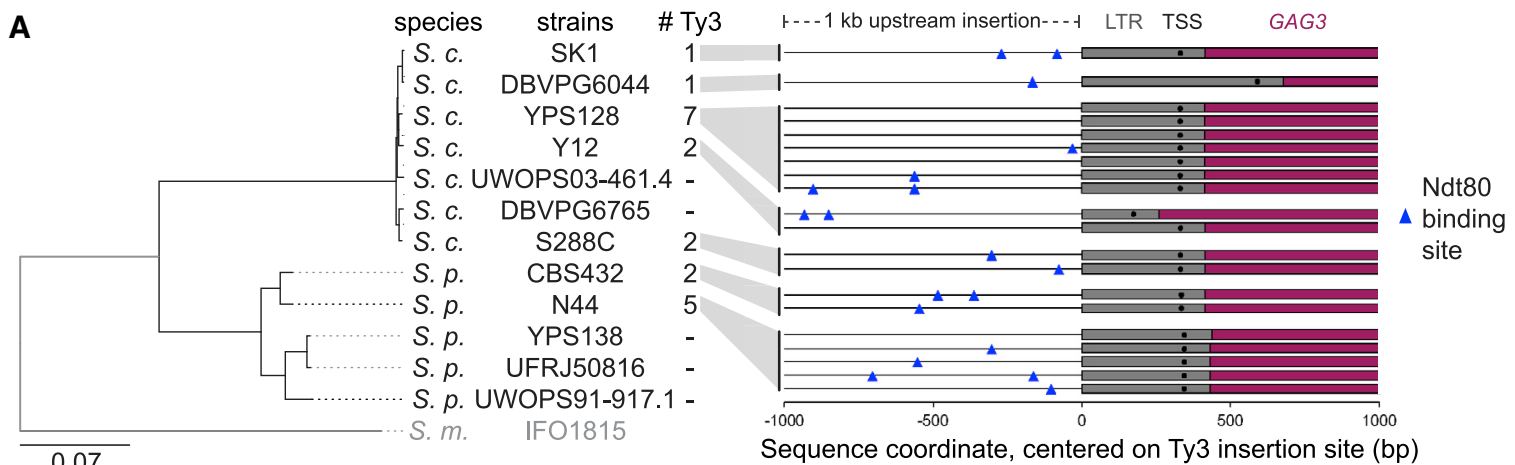

\section{B}

Ndt80 target genes in SK1 $(n=260)$
- Ndt80 target -

non-Ndt80 target genes in SK1 $(n=5291)$ non-target

All Ty3/Gypsy in 13 genomes $(n=518)$ full-length and solo-LTRs

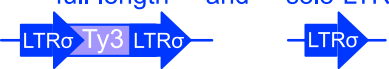

Distribution Ndt80 motif:

1000 bootstraps

mean \pm SD

outlier boundaries

1000 bootstraps
mean \pm SD
outlier boundaries

_ observed

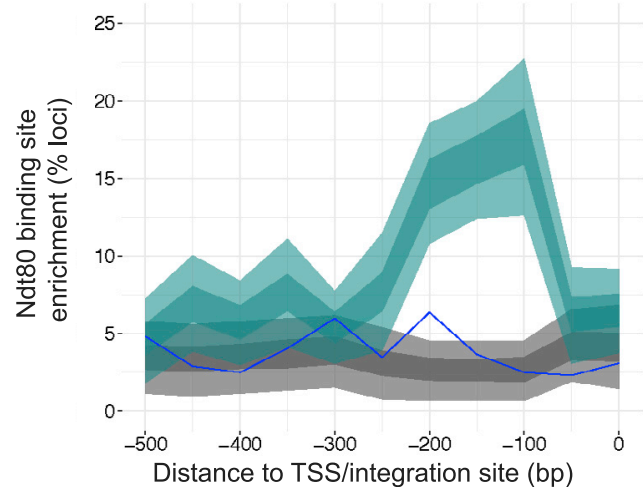

Figure 3. Integration of Ty3 Downstream of Ndt80 Is a Recurring Phenomenon

(A) Left: phylogenetic relationships among seven S. cerevisiae (S. c.) strains and five S. paradoxus (S. p.) strains (Modified from Yue et al., 2017), along with their respective number of full-length Ty3 elements (total 20). Right: 2-kb window map of 1,000 bp upstream of full-length Ty3 elements with Ndt80 binding sites (CACAAA) indicated as blue triangles. Ndt80 binding sites are significantly enriched in this region (determined by $Z$ test statistic of 100 randomized simulations; $Z$ score 2.7; $\mathrm{p}<0.01)$.

(B) Left: 260 SK1 genes were identified as Ndt80 target genes (Chu et al., 1998), and 5,291 as non-target genes. Within the 13 S.c. and S.p. strains analyzed above there are 518 Ty3 elements (20 full-length and 498 solo LTRs). Right: Comparison of the distribution of Ndt80 binding sites in the region 500 bp upstream of the TSS among target genes (teal), non-target genes (gray), and Ty3 elements (blue). Statistical significance was determined by bootstrap analysis ( $<<0.001$ at the -200 position).

\section{Meiotic Ty3 Repression Is Mediated by the Translational Repressor Rim4}

We next sought to elucidate the mechanism of $T y 3$ translational repression. Translational control of mRNA plays a central role in regulating gene expression during gametogenesis in virtually all sexual organisms (reviewed in Kronja and Orr-Weaver, 2011). In most organisms, transcription is shut off or limited during meiosis and regulation of gene expression relies on RNA-binding proteins that govern translation of previously transcribed mRNA. In S. cerevisiae, the RNA-binding protein Rim4 binds to and inhibits translation of mRNAs that encode protein products that are toxic when prematurely expressed but are necessary for late meiotic events (Berchowitz et al., 2013). We thus wanted to test whether repression of Ty3 translation was mediated by Rim4. Because Rim4 is required for meiotic entry, we designed a selectively degradable Rim4 using the auxin-inducible degron system (Morawska and Ulrich, 2013) such that we could deplete it just before activation of NDT80. We found that Ty3 was translated upon premature Rim4 depletion (Figure 5). Rim4 must be assembled into an amyloid-like state in order to repress translation of its targets, which requires the intrinsically disordered region (IDR) in its $\mathrm{C}$ terminus (Berchowitz et al., 2015). We found that rim4 $4 I D R$ mutants also translate Ty3 (Figure S5). While our genetic analyses do not allow us to distinguish whether Rim4 directly or indirectly regulates $T y 3$ translation, we conclude that Rim4 amyloid-like repressors function in part to protect the germline from the proliferation of retrotransposons.

\section{Mammalian Gypsy-Derived Genes Are Regulated by} Meiotic Transcription Factors

We next asked whether mammalian Ty3/Gypsy-like elements also exploit meiosis-specific transcription factors to activate during gametogenesis. In mammals, most Ty3/Gypsy LTR-retrotransposon/retrovirus-derived elements no longer retain their retrotransposition capacity and are classified based on whether Gypsy-internal sequences are present (28 loci in mouse and 693 loci in human contain these sequences) (Campillos et al., 2006; Kojima, 2018). To test if any mammalian Gypsy-derived genes are switched on during meiosis, we analyzed RNA-seq data from adult mouse testes (Soumillon et al., 2013). We found that several Gypsy-derived elements, including those that contain Gag-like internal sequence (MamGyp-int), are transcribed in testes during the meiotic stage of spermatogenesis (spermatocytes) and in postmeiotic round spermatids (Figure S6A). When we analyzed uniquely mapping RNA-seq reads of specific MamGyp-int loci, we found that two genes-Moap1 and Pnma1-are 
A
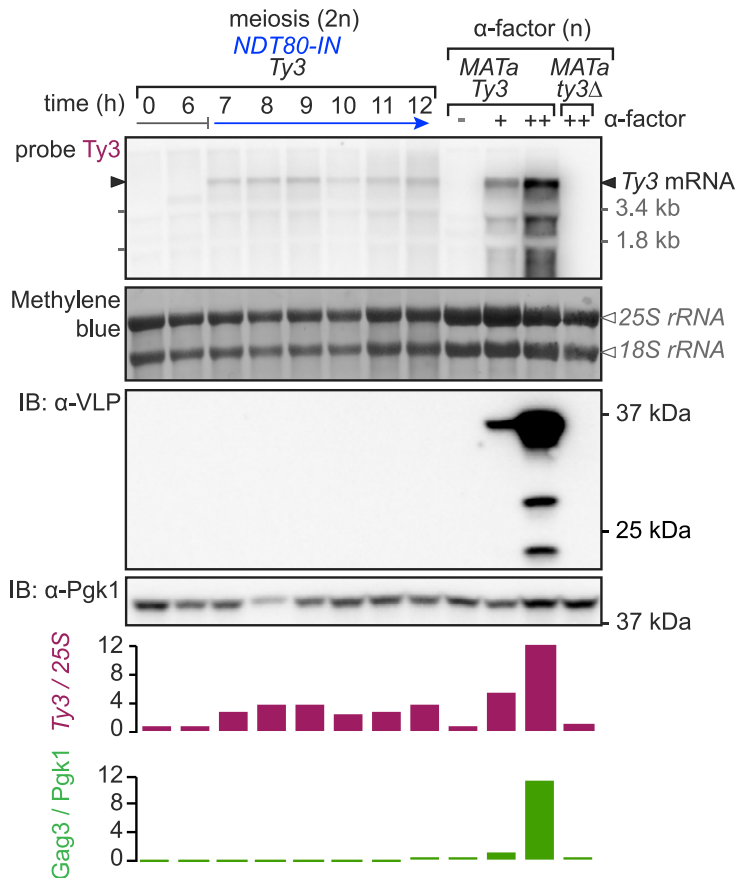

B

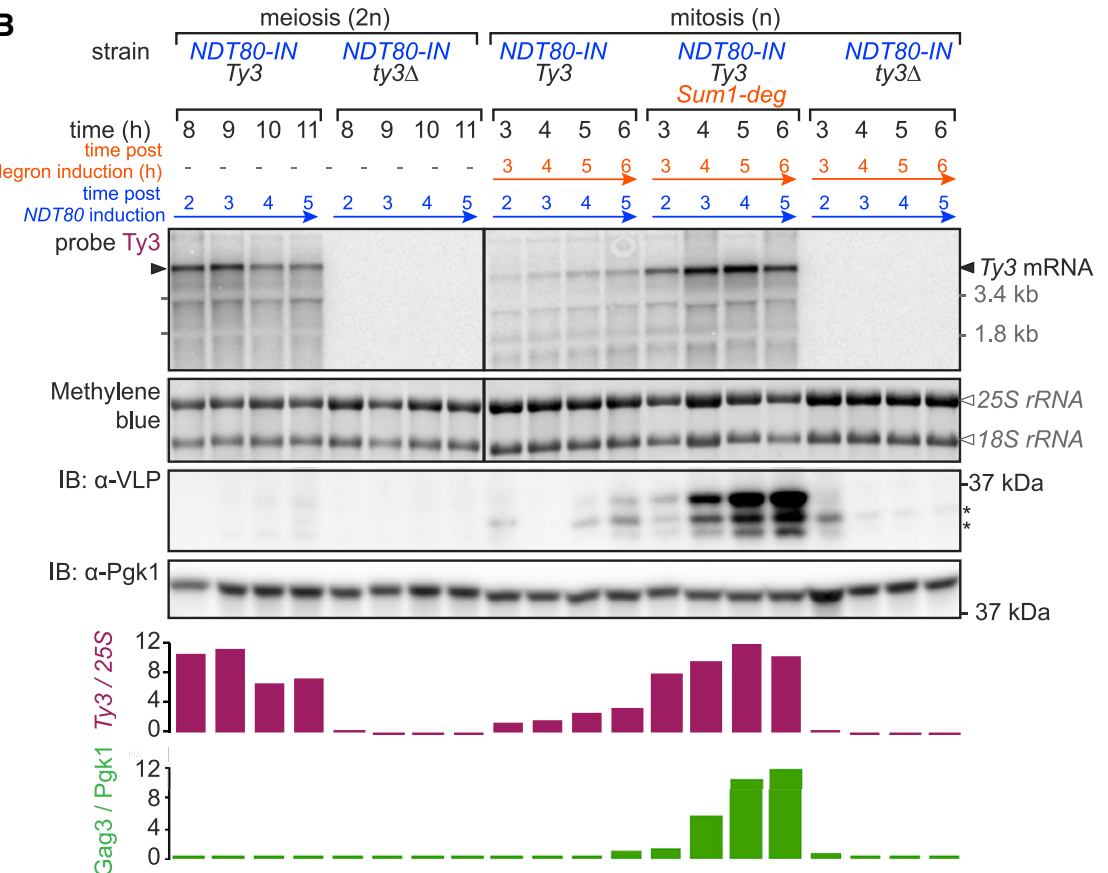

Figure 4. Тy 3 Is Post-Transcriptionally Repressed in Meiosis

(A) Gag3 protein encoded by Ty3 is not detectable during meiosis. Sporulation was induced in NDT80IN (B48) as in 2A. MATa wild-type (B1) and ty3 4 (B827) haploid strains were exposed to $\alpha$-factor $(0$, $0.5,10 \mu \mathrm{M}$ ) to induce Ty3 activation. Left: Ty3 mRNA (black arrow) and $r R N A$ (white arrows) levels, and Gag3 (and its processed forms CA-SP, CA) and Pgk1 (loading control) protein levels are shown. Migration of molecular size standards is indicated on right as kDa values. Right: Quantification of Ty3 fulllength RNA (maroon) and Gag3 protein (green) normalized over their respective loading controls. Shown are representative results of $\geq 5$ biological replicates.

(B) Post-transcriptional repression of Gag3 is specific to meiosis. To express Ty 3 in both meiosis and mitosis, we expressed NDT80 in sporulating diploid cells (wild-type B48, ty3د B839) and in haploid mitotically dividing cells with and without the Sum1 transcriptional repressor (wild-type B477, Sum1deg B829, and ty3 $\triangle$ B827). Ty3 mRNA (black arrow) and $r R N A$ (white arrows) levels and Gag3 and Pgk1 (loading control) protein levels and quantification are shown, as in (A). Asterisks show unspecific bands (e.g., band in $\alpha$-VLP present in ty $3 \Delta$; see also Figure S3B). Shown are representative results of 5 biological replicates.

supials that did not experience this expansion of Pnma genes and harbor only a single Pnma gene: M-PNMA (Kokošar and Kordiš, 2013). We analyzed tissue-specific RNA-seq data from opossum (Lesch et al., 2016; Marin et al., 2017) and found that $M$ PNMA mRNA is highly and specifically upregulated in testes, particularly in pachytene spermatocytes (Figure S6B). These data suggest that meiotic transcription of Pnma was either present in the common therian ancestor or has convergently evolved independently in both eutherians and marsupials.

We next wished to investigate if the transcriptional activation of Gypsy-derived elements in meiosis reflects the capture of element-external meiosis-specific transcription factor binding sites. Motivated by our findings in yeast, we focused our investigation on two master transcriptional

highly upregulated in mouse meiotic spermatocytes (Figure 6A). Both Moap 1 and Pnma1 are characterized as domesticated in mammals, and their open reading frames encode Gypsy-derived Gag-like proteins. Based on these analyses, we propose that, analogous to Ty3 regulation in yeast, the Gypsy-like sequences Moap 1 and Pnma1 are being transcriptionally activated in male mouse meiosis. In eutherian mammals, there has been a massive expansion of the Pnma gene family (15 genes in humans, 12 in mice; Campillos et al., 2006). We wanted to test whether meiotic transcription of Pnma can be observed in mar- regulators of meiosis - Stra8 and Mybl1 (also known as A-myb). Stra8 is required for meiotic initiation in mammals and promotes transcription of early meiotic genes (Anderson et al., 2008), whereas Mybl1 becomes active early in meiosis and is required for meiosis to progress through pachytene (Bolcun-Filas et al., 2011). We assessed whether the meiotic transcription factors Stra8 and Mybl1 bind upstream of Moap1 and/or Pnma1 using ChIP-seq data from adult mouse testes (Kojima et al., 2019; Zhou et al., 2017). We found that both Mybl1 and Stra8 ChIPseq peaks are present upstream of multiple Moap1 TSSs 


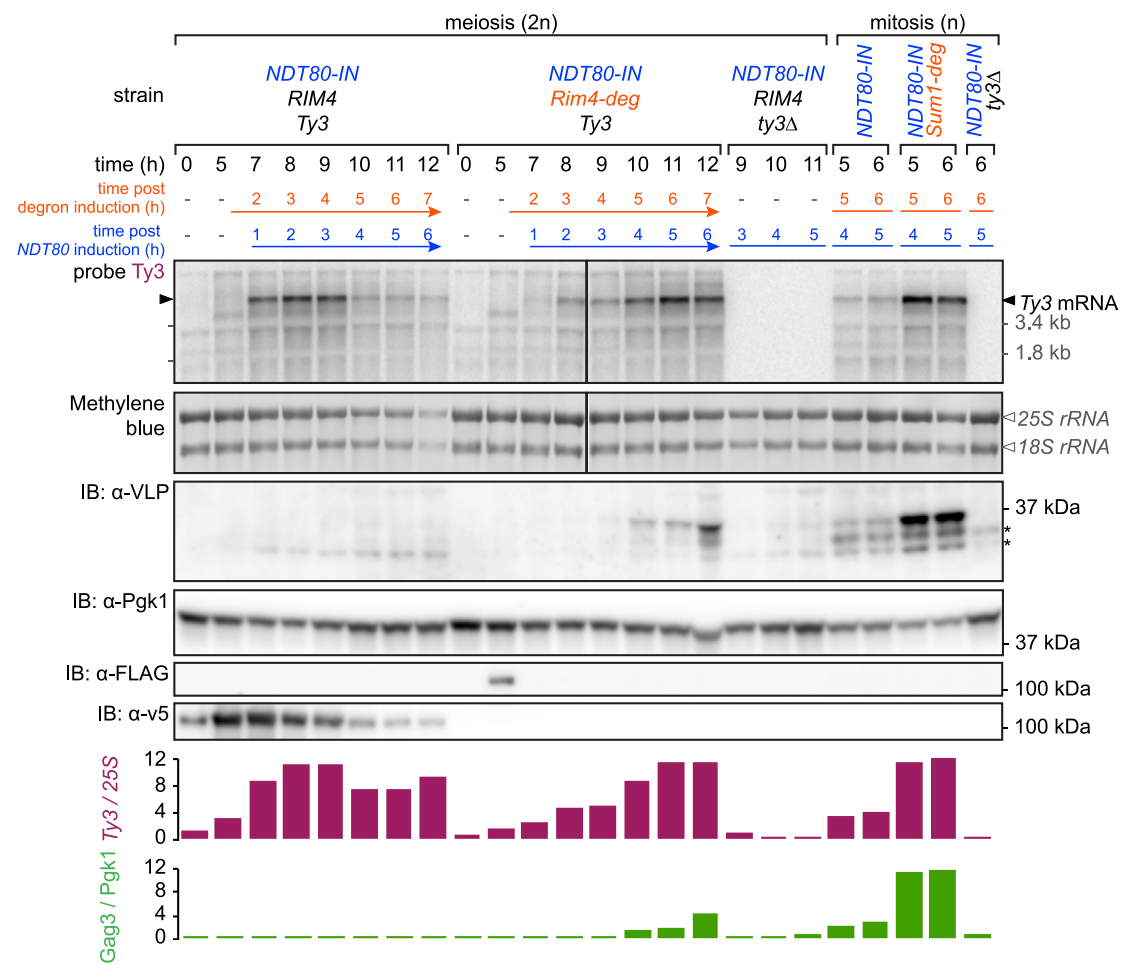

Figure 5. Ty3 Is Translationally Repressed by the RNA-Binding Protein Rim4

Meiosis was induced in three NDT80-IN diploid strains: wild-type (B48), Rim4-deg (B1022, Rim4degron fusion strain, induced by auxin addition to $1 \mathrm{mM}$ at $\mathrm{t}=5 \mathrm{~h}$ ), and ty $3 \Delta$ (B389). Meiotic cells were released from the $\mathrm{G} 2$ block by addition of $1 \mu \mathrm{M}$ $\beta$-estradiol at $6 \mathrm{~h}$. Mitotic samples were collected from the experiment shown in $3 \mathrm{~B}$ and Figure S3B. Ty3 mRNA (black arrow) and rRNA (white arrows) levels and Gag3, Rim4 (Rim4-deg is tagged with FLAG, wild-type Rim4 is tagged with $3 \mathrm{~V} 5$ in the first strain, and not tagged in the remaining strains), and Pgk1 (loading control) protein levels. Migration of molecular size standards is indicated on right as $\mathrm{kDa}$ values. Quantification of Ty3 mRNA and Gag3 protein relatively to their respective loading controls are shown. Asterisks show unspecific bands (e.g., band in $\alpha$-VLP present in ty $3 \Delta$; see also Figure S3B). Shown are representative results of 4 biological replicates.
(Figures 6B, S6C, and S6D). Similarly, a Mybl1 ChIP-seq peak overlaps the Pnma1 TSS external to the MamGyp-int sequence (Figures 6B, S6C, and S6D).

If Moap 1 and Pnma1 are under the direct control of Stra8 and Mybl1, developing gametes that do not express these transcription factors should not produce these transcripts. To test this prediction, we analyzed whether Moap1 and Pnma1 are expressed in preleptotene $\mathrm{Stra8}^{+/-}$and preleptotene Stra8 ${ }^{-/-}$ spermatocytes (Kojima et al., 2019) or in P14 Myb/1 ${ }^{+/-}$and Myb/1 ${ }^{-1-}$ testes (Zhou et al., 2017). In concordance with the ChIP-seq analysis, Moap1 transcript abundance was significantly reduced in the absence of Stra8 (Figure 6D). In addition, both Pnma1 and Moap1 transcript abundance was reduced in the absence of Mybl1 (Figure 6E). Taken together, our data support the hypothesis that Gypsy elements co-opt critical meiotic transcription factors to ensure their expression in gametogenesis and that this regulation exists from yeast to mammals.

\section{Mammalian Meiotic Translational Repressor Proteins Bind to GAG-Containing mRNA}

In mammals, the functional orthologs of Rim4 are the DAZ family of RNA-binding proteins (Berchowitz et al., 2015). DAZ family proteins act as regulators of translation and exhibit a similar organization to Rim4 in that they harbor N-terminal RRMs and a C-terminal prion-like domain (King et al., 2012). Furthermore, Dazl (DAZ-like) forms SDS-resistant amyloid-like assemblies specifically in mouse testes (Berchowitz et al., 2015). Thus, we asked if meiotic Gypsy-derived RNAs might be regulated by DAZ family proteins. We analyzed Dazl CLIP data generated from adult mouse testes (Zagore et al., 2018) and observed clear enrichment peaks within the $3^{\prime}$ UTRs of Moap1 and Pnma1 transcripts (Figures 6B and 6C). In addition, we analyzed DAZL-RNA significantly enriched in DAZL immunoprecipitations (Figures $6 \mathrm{~F}$ and $\mathrm{S} 6 \mathrm{E})$. Of all the 802 different types of retrotransposon expressed in this tissue, only five were enriched in the DAZL-RNA immunoprecipitations (Figures S6F and S6G). We propose that the physical interaction between DAZ family proteins and transcripts containing Gypsy-derived sequences exists in both mice and humans and in both male and female gametogenesis. Taken together, these data support an ancient association between amyloid-like RNA-binding assemblies and retrotransposon and retrotransposon-derived mRNA. We propose that this association possibly represents a functional repurposing of the retrotransposon defense mechanism we observed in yeast.

\section{DISCUSSION}

In this study, we elucidate mechanisms underlying an evolutionary battlefront, where retrotransposons attempt to proliferate in the germline and host organisms respond to defend the genomes of their progeny. Ty3/Gypsy retrotransposons in yeast and domesticated Gypsy-derived genes in mammals co-opt host master meiotic regulators to transcriptionally activate during gametogenesis. Based on these findings, we propose that the integration site of a retrotransposon can encode the critical regulatory information that the element requires to activate specifically during gamete development. We further show that yeast cells utilize a meiosisspecific translational repressor to thwart further progress in the retrotransposon life cycle. Likewise, transcripts from domesticated Ty3/Gypsy elements in mammals are bound by a cognate translational regulator. In the yeast strain SK1, we find that meiotic cells efficiently repress translation of Ty3 mRNA, which very likely prevents the element from proliferating in this context. However, because the control of retrotransposons by meiotic transcription 
A

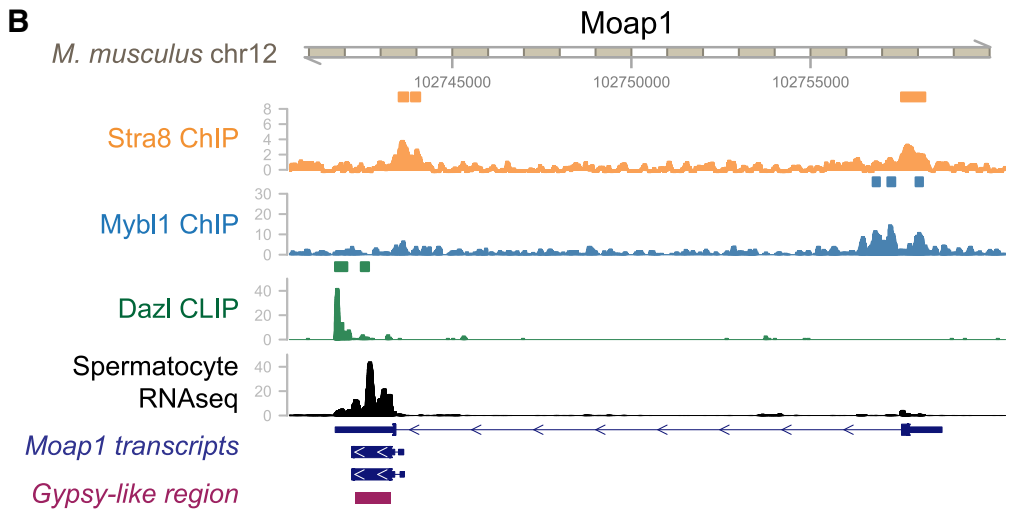

C
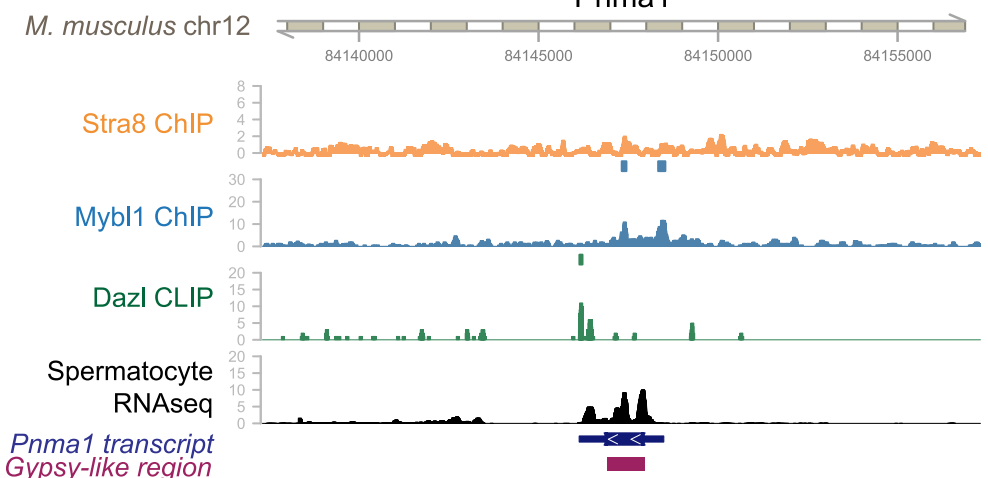

D

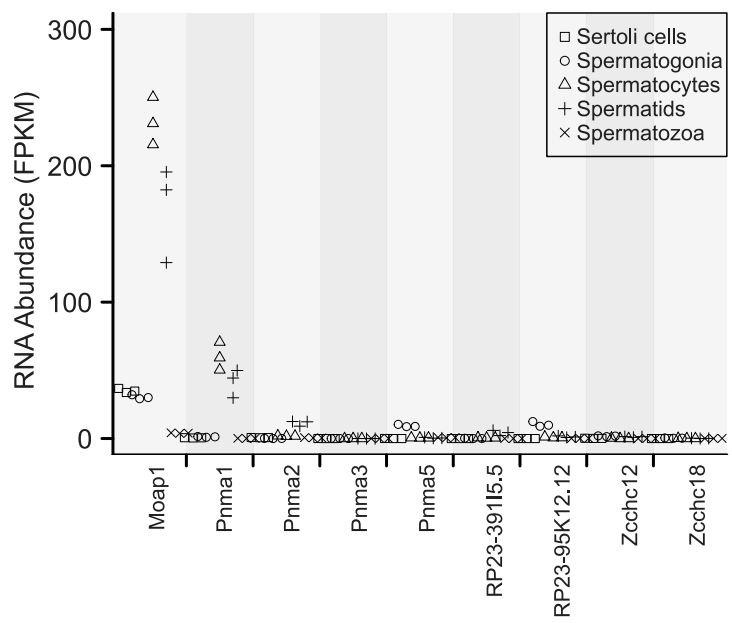

B

Pnma1

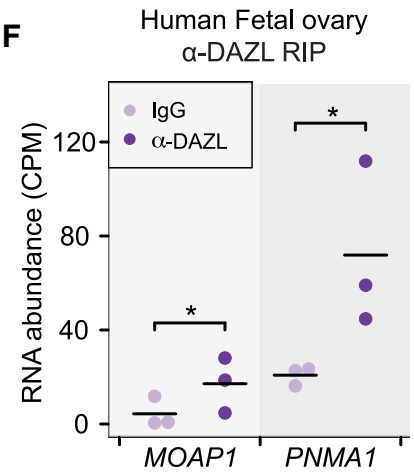

Figure 6. Mammalian Gypsy-Derived Genes Are Activated by Meiotic Transcription Factors and Regulated by Meiotic RNA-Binding Proteins (A) Analysis of uniquely mapping MamGyp-int RNA-seq reads for MamGyp-int loci expressed during spermatogenesis in adult mouse testes. biological replicates $=3$ for each stage.

(B and C) Browser tracks showing mouse testis Stra8-FLAG ChIP-seq peaks (orange bars, MACS2 q $<0.05$ ), mouse testis Stra8-FLAG ChIP reads (orange line plot, fold change in ChIP relative to input, data from wild-type animals subtracted as background), mouse testis Mybl1 ChIP-seq peaks (blue bars, MACS2 $\mathrm{q}<$ 0.05), mouse testis Mybl1 ChIP reads (blue line plot, fold change relative to input), mouse testis Dazl CLIP clusters (green bars, biologically reproducible CLIP clusters), mouse testis Dazl CLIP (green line plot, number of CLIP tags), mouse spermatocyte RNA-seq (black histogram, counts per million mapped reads), Gencode VM18 gene models encompassing (B) Moap1, or (C) Pnma1 (navy rectangles), and the genomic location of Gypsy-derived sequences (maroon rectangles).

(D) Expression of Moap1 and Pnma1 in Stra8 ${ }^{-/-}$preleptotene mouse spermatocyte RNA-seq data. CPM (counts per million) mapped reads (biological replicates $=2$ for Stra8 ${ }^{+/-}$spermatocytes and $=3$ for Stra8 ${ }^{-1-}$ spermatocytes).

(E) Expression of Moap1 and Pnma1 in P14 Myb/1 ${ }^{-1-}$ mouse testis RNA-seq data (biological replicates = 3).

(F) Analysis of MamGyp-int containing transcripts from MOAP1 and PNMA1 in anti-DAZL RIP from human fetal ovaries (biological replicates = 3).

Asterisks indicate $\mathrm{p}<0.05$ as determined by Student's $\mathrm{t}$ test. 
factors occurs frequently in yeast, we expect this activation strategy to be successful in other strains and other organisms. Future studies will be necessary to determine the extent to which meiotic activation and host translational repression of Ty3/Gypsy elements can be generalized across organisms.

Arkhipova and Meselson put forth the idea that one of the major forces driving the pervasive maintenance of meiosis and sex is to allow organisms to focus defense strategies against transposable elements to a subset of cells (i.e., the germline) that will provide the genetic material for generations to come (Arkhipova and Meselson, 2005). In multicellular organisms, it is of obvious benefit to the retrotransposon to direct activation to the germline with the aim to elude host defense mechanisms. Why does the Ty3 retrotransposon activate only during the sexual cycle (i.e., meiosis and mating) in a unicellular organism such as yeast? In unicellular organisms, the entire organism is the germline, and thus, coordination of retrotransposon activation with meiosis may seem less obvious. Retrotransposon activation poses a resource cost on the cell (reviewed in Bourque et al., 2018). In rapidly proliferating cells, this cost could indeed translate into a fitness disadvantage. When activation is restricted to meiosis and mating in yeast, Ty 3 remains latent, thus minimizing its fitness cost and focusing its efforts to proliferate in cells that are not cycling. Furthermore, linking retrotransposon proliferation to meiotic recombination (i.e., mixis) could be valuable for the element. Organisms can generate favorable allelic combinations via meiotic recombination. Retrotransposons could propagate into advantageous allelic combinations by activating during and post-recombination, which could offset the fitness cost of an additional element. Lastly, the programmed double-strand breaks that initiate meiotic recombination could provide access points for retrotransposon cDNA.

The transcriptional activation of LTR-retrotransposons is often governed by regulatory sequences within the LTRs themselves. For example, the $5^{\prime}$ LTR of Ty1/Copia contains binding sites for several transcriptional activators, including Gcn4, Gcr1, Ste12, Tec1, and Tea1, as well as the repressor Mot3 (Servant et al., 2008). Likewise, the ability of Ty3/Gypsy to activate during mating (or upon $\alpha$-factor exposure) relies on Ste12 binding within its 5' LTR (Bilanchone et al., 1993). However, in the situation described here, Ty3 utilizes regulatory sequences upstream of its integration site. What selective pressures would then drive Gypsy-like elements to utilize external regulatory sites to activate during meiosis? We propose that there may be some advantages to decouple meiotic activation from the intrinsic properties of the element. Meiosis-specific genes are repressed during vegetative-somatic growth. As we mentioned above, in yeast, the Ndt80 binding site is also recognized by the repressor Sum1 (Klutstein et al., 2010; Pierce et al., 2003). If this sequence was harbored in the $5^{\prime}$ LTR, the element could be condemned to a single strategy by virtue of the presence of Sum1 in nonmeiotic contexts. By utilizing external regulatory elements, the element can avoid intrinsic repressor binding sites while gaining the flexibility to utilize other transcription activators that may be advantageous in other selective contexts.

As illustrated in decades of previous work, the study of Ty elements is a powerful model for the discovery and elucidation of host mechanisms that restrict retrotransposition (Maxwell and Curcio, 2007). Genetic screens designed to identify factors that influence Ty1 (Griffith et al., 2003; Scholes et al., 2001) and Ty3 (Aye et al., 2004; Irwin et al., 2005) retrotransposition have shown that RNA processing factors can positively and negatively influence Ty retrotransposition. Three RNA processing factors suppress both Ty1 and Ty3 retrotransposition: the DExH-box RNA helicase $\mathrm{Dbp} 3$, the ribosomal protein Rpl6A, and the lariat-debranching enzyme Dbr1. Studies motivated, in part, from these screens have identified that mRNA-processing bodies ( $P$ bodies) (Beliakova-Bethell et al., 2006) and nucleoporins (Rowley et al., 2018) play important roles in the life cycle of Ty3 elements. Here, we show that host repression of Ty3 is either directly or indirectly carried out by amyloid-like assemblies of the RNAbinding protein Rim4. In future studies, it will be exciting to assess whether and how Rim4 assemblies interact with these factors to defend the host genome against retrotransposon proliferation.

Rim4 binds to and represses the translation of numerous mRNAs that encode proteins required for late meiotic events. While many of these targets are translated precisely at meiosis II onset, several others are translated later (Berchowitz et al., 2013; Brar et al., 2012) and apparently some, such as Ty3, are seemingly never translated. We hypothesize that the fate of Rim4 targets is dictated by interaction with other factors. This hypothesis is consistent with the developmentally regulated clearance of Rim4 multimeric assemblies at meiosis II onset (Carpenter et al., 2018). Targets that are translated significantly after meiosis II onset likely interact with the RNA-binding proteins Pes4 and Mip6 (Jin et al., 2017), and we speculate that the terminal repression we observe for Ty 3 is carried out by a factor unknown to us.

To escape Rim4-mediated translational repression, Ty3 could take advantage of a particular feature of the meiotic stage at which it is expressed. Prior to meiotic prophase exit, cells can return to a mitotic cycle if nutrients are sensed by the cell in a process known as "return to growth," which is a common event in the life cycle of wild yeast (Brion et al., 2017; Tsuchiya et al., 2014). Ty3 mRNA accumulates 15-30 min before the meiotic commitment point. Upon return to growth, Rim4-mediated translational control is relieved (Jin et al., 2017), and, therefore, Ty3 would recover the ability to propagate in a post-mixis environment. Similarly, if Ty3 mRNA persists until the end of gametogenesis and into spores, germination would likely provide a permissive environment for Ty3 propagation. This strategy would be analogous to what is observed in LINE-1 elements, which are expressed during mouse gametogenesis and then only reintegrate after fertilization during early embryonic development (Kano et al., 2009).

While the proliferation of Ty3/Gypsy elements is often deleterious, it is possible that their evolutionary cost is offset by providing an activity or function that is beneficial to the host (Cosby et al., 2019). Our preliminary results suggest that Ty3 deletion mutants do not exhibit a detectable defect (or advantage) in any aspect of gametogenesis, including meiosis, spore formation, and/or spore viability. However, we speculate that mutations in other Ty elements could be different in this regard. One exciting speculation is that, by integrating downstream of meiosis-specific regulatory elements, a new coding sequence is now readily available to be domesticated for a meiosis-specific function. Similarly, the interaction of retrotransposons with 
regulatory RNA-binding proteins could connect the evolving gene to additional regulatory pathways. Retrotransposons are ripe for domestication because they encode several proteins with diverse and useful domains (Volff, 2006). Possible examples of meiotic genes evolving from Gypsy sequences include the mammalian Gypsy-like elements Moap1 and Pnma1, which do not represent active retrotransposons and are likely domesticated. We observe meiotic transcriptional activation of Pnma genes in mouse, human, and opossum, and while meiosis-specific activation of Gypsy-like elements is unlikely to be generalizable among all copies of these elements within a species, it appears to be generalizable to some degree among species. Remarkably, the single Pnma gene (M-PNMA) in opossum is highly upregulated in pachytene spermatocytes. Whether this represents the ancestral expression pattern of Pnma or convergent evolution, we speculate that meiotic activation of Pnma is providing some benefit to the organism and is being selected for. Because Pnma is inactive for retrotransposition, we propose that during the time when Gypsy elements were still retrotranspositionally active and co-opting meiotic transcription factors for their own benefit, they acquired beneficial functions in the host meiotic cells. It is exciting to us that the regulatory circuitry established during the evolutionary battle between host and retrotransposon could also give direction as to how and when the emerging gene will function. It will be important to investigate the emerging roles of these elements and how they are repurposing retrotransposon regulatory mechanisms in the process of acquiring functions and evolving into new genes.

We propose that the co-opting of meiosis-specific transcription factors by retrotransposons represents an ancient strategy to coordinate retrotransposition with germline development. Correspondingly, translational repression of retrotransposons by host RNA-binding proteins could also be an ancient and preserved feature of meiosis. We can add this mechanism to the elaborate repertoire of retrotransposon defense systems such as piRNA, DNA methylation, and heterochromatic DNA condensation. It will be interesting to assess whether other assemblyforming RNA-binding proteins intervene in the life cycles of other retrotransposons in other organisms.

\section{STAR $\star$ METHODS}

Detailed methods are provided in the online version of this paper and include the following:

- KEY RESOURCES TABLE

- RESOURCE AVAILABILITY

O Lead Contact

- Materials Availability

- Data and Code Availability

- EXPERIMENTAL MODEL AND SUBJECT DETAILS

Y Yeast Strains and Constructs

- METHOD DETAILS

Y Yeast Growth and Sporulation Conditions

- Meiotic Progression Analysis

O Northern Blot Analysis

- Yeast RNA Sequencing and Sequencing Analysis

Immunoblot Analysis

Ndt80 ChIP-seq
- QUANTIFICATION AND STATISTICAL ANALYSIS

O Statistical Analysis for Ndt80 Binding Site Enrichment Upstream Ty3 Insertions

O Analysis of Yeast ChIPseq Data

O Analysis of Mammalian RNAseq Data (Mouse, Opossum)

O Analysis of Mammalian ChIPseq Data (Mouse)

$\bigcirc$ Analysis of DazI CLIP and DAZL RIP Mammalian Data (Mouse, Human)

\section{SUPPLEMENTAL INFORMATION}

Supplemental Information can be found online at https://doi.org/10.1016/j. devcel.2020.11.008.

\section{ACKNOWLEDGMENTS}

We would like to thank Virginia Bilanchone and Suzanne Sandmeyer for providing the anti-Ty3 VLP crude serum, Kayla Carpenter for RNA-sequencing prep, and S. Grace Herod for the his3::pRIM4-OsTIR1::His3MX6 construct. We would like to thank Albert Serra Cardona and Zhiguo Zhang for help with Ndt80 ChIP. We would also like to thank Stephen Goff and members of the Berchowitz lab for valuable discussions and Jonathan Dworkin, Diana Ottoz, and Tanguy Lucas for critical reading of the manuscript. This research is supported by the Schaefer Research Scholars Program, the Hirschl Family Trust, NIH grant R35 GM124633-01 to L.E.B., MRC University Unit grant MC_UU_00007/6 to I.R.A., Agence Nationale de la Recherche grant ANR-16-CE12-0019 to G.L. and National Institutes of Health DP2 AG055946-1250 01 to E.Ü.; Finally, we would like to thank Harmit Malik who suggested an idea to L.E.B. that provided the motivation to begin this project.

\section{AUTHOR CONTRIBUTIONS}

L.E.B. and R.L. conceived of the study. L.E.B. and R.L. designed the molecular biology and yeast computational experiments. I.R.A. designed and analyzed the mammalian computational biology experiments. R.L. led the experimental procedures. R.L., A.D., G.D., S.B., H.A., E.U., A.H., and L.E.B. built strains, performed experiments, and analyzed the data. G.L., J.-X.Y., and M.D.C. screened the wild yeast strains for sporulation efficiency and estimated Ty count. L.E.B. and R.L. wrote the paper with input from all authors. The manuscript was edited by I.R.A., E.U., and G.L.

\section{DECLARATION OF INTERESTS}

The authors declare no competing interests.

Received: March 27, 2020

Revised: June 25, 2020

Accepted: November 6, 2020

Published: December 4, 2020

\section{REFERENCES}

Anders, S., Pyl, P.T., and Huber, W. (2015). HTSeq-a Python framework to work with high-throughput sequencing data. Bioinformatics 31, 166-169.

Anderson, E.L., Baltus, A.E., Roepers-Gajadien, H.L., Hassold, T.J., de Rooij, D.G., van Pelt, A.M.M., and Page, D.C. (2008). Stra8 and its inducer, retinoic acid, regulate meiotic initiation in both spermatogenesis and oogenesis in mice. Proc. Natl. Acad. Sci. USA 105, 14976-14980.

Arkhipova, I., and Meselson, M. (2005). Deleterious transposable elements and the extinction of asexuals. BioEssays 27, 76-85.

Aye, M., Irwin, B., Beliakova-Bethell, N., Chen, E., Garrus, J., and Sandmeyer, S. (2004). Host factors that affect Ty3 retrotransposition in Saccharomyces cerevisiae. Genetics 168, 1159-1176.

Bao, J., and Yan, W. (2012). Male germline control of transposable elements. Biol. Reprod. 86, 162, 1-162,14. 
Developmental Cell Article

Barau, J., Teissandier, A., Zamudio, N., Roy, S., Nalesso, V., Hérault, Y., Guillou, F., and Bourc'his, D. (2016). The DNA methyltransferase DNMT3C protects male germ cells from transposon activity. Science 354, 909-912.

Beliakova-Bethell, N., Beckham, C., Giddings, T.H., Winey, M., Parker, R., and Sandmeyer, S. (2006). Virus-like particles of the Ty3 retrotransposon assemble in association with P-body components. RNA 12, 94-101.

Benjamin, K.R., Zhang, C., Shokat, K.M., and Herskowitz, I. (2003). Control of landmark events in meiosis by the CDK Cdc28 and the meiosis-specific kinase Ime2. Genes Dev. 17, 1524-1539.

Berchowitz, L.E., Gajadhar, A.S., van Werven, F.J., De Rosa, A.A., Weissman, J.S., Samoylova, M.L., Brar, G.A., Xu, Y., Xiao, C., Futcher, B., et al. (2013). A developmentally regulated translational control pathway establishes the meiotic chromosome segregation pattern. Genes Dev. 27, 2147-2163.

Berchowitz, L.E., Kabachinski, G., Walker, M.R., Carlile, T.M., Gilbert, W.V., Schwartz, T.U., and Amon, A. (2015). Regulated formation of an amyloid-like translational repressor governs gametogenesis. Cell 163, 406-418.

Bilanchone, V.W., Claypool, J.A., Kinsey, P.T., and Sandmeyer, S.B. (1993). Positive and negative regulatory elements control expression of the yeast retrotransposon Ty3. Genetics 134, 685-700.

Bolcun-Filas, E., Bannister, L.A., Barash, A., Schimenti, K.J., Hartford, S.A. Eppig, J.J., Handel, M.A., Shen, L., and Schimenti, J.C. (2011). A-MYB (MYBL1) transcription factor is a master regulator of male meiosis. Development 138, 3319-3330.

Bourc'his, D., and Bestor, T.H. (2004). Meiotic catastrophe and retrotransposon reactivation in male germ cells lacking Dnmt3L. Nature 431, 96-99.

Bourque, G., Burns, K.H., Gehring, M., Gorbunova, V., Seluanov, A., Hammell, M., Imbeault, M., Izsvák, Z., Levin, H.L., Macfarlan, T.S., et al. (2018). Ten things you should know about transposable elements. Genome Biol. 19, 199.

Brar, G.A., Yassour, M., Friedman, N., Regev, A., Ingolia, N.T., and Weissman, J.S. (2012). High-resolution view of the yeast meiotic program revealed by ribosome profiling. Science 335, 552-557.

Brion, C., Legrand, S., Peter, J., Caradec, C., Pflieger, D., Hou, J., Friedrich, A., Llorente, B., and Schacherer, J. (2017). Variation of the meiotic recombination landscape and properties over a broad evolutionary distance in yeasts. PLoS Genet. 13, e1006917.

Campillos, M., Doerks, T., Shah, P.K., and Bork, P. (2006). Computational characterization of multiple Gag-like human proteins. Trends Genet. 22, 585-589.

Carlile, T.M., and Amon, A. (2008). Meiosis I is established through divisionspecific translational control of a cyclin. Cell 133, 280-291.

Carpenter, K., Bell, R.B., Yunus, J., Amon, A., and Berchowitz, L.E. (2018). Phosphorylation-mediated clearance of amyloid-like assemblies in meiosis. Dev. Cell 45, 392-405.e6.

Chu, S., Derisi, J., Eisen, M., Mulholland, J., Botstein, D., Brown, P.O., and Herskowitz, I. (1998). The transcriptional program of sporulation in budding yeast. Science 282, 699-705.

Chuong, E.B., Elde, N.C., and Feschotte, C. (2017). Regulatory activities of transposable elements: from conflicts to benefits. Nat. Rev. Genet. 18, 71-86. Cosby, R.L., Chang, N.C., and Feschotte, C. (2019). Host-transposon interactions: conflict, cooperation, and cooption. Genes Dev. 33, 1098-1116.

Crichton, J.H., Dunican, D.S., MacLennan, M., Meehan, R.R., and Adams, I.R. (2014). Defending the genome from the enemy within: mechanisms of retrotransposon suppression in the mouse germline. Cell. Mol. Life Sci. 71, 1581-1605.

Cristofari, G., Gabus, C., Ficheux, D., Bona, M., Le Grice, S.F.J., and Darlix, J.L. (1999). Characterization of active reverse transcriptase and nucleoprotein complexes of the yeast retrotransposon Ty3 in vitro. J. Biol. Chem. 274, 36643-36648.

De Muyt, A., Pyatnitskaya, A., Andréani, J., Ranjha, L., Ramus, C., Laureau, R., Fernandez-Vega, A., Holoch, D., Girard, E., Govin, J., et al. (2018). A meiotic XPF-ERCC1-like complex recognizes joint molecule recombination intermediates to promote crossover formation. Genes Dev. 32, 283-296.
Dechaud, C., Volff, J.N., Schartl, M., and Naville, M. (2019). Sex and the TEs: transposable elements in sexual development and function in animals. Mob. DNA 10, 42.

Descrimes, M., Ben Zouari, Y., Wery, M., Legendre, R., Gautheret, D., and Morillon, A. (2015). VING: a software for visualization of deep sequencing signals. BMC Res. Notes 8, 419

Dobin, A., Davis, C.A., Schlesinger, F., Drenkow, J., Zaleski, C., Jha, S., Batut, P., Chaisson, M., and Gingeras, T.R. (2013). STAR: ultrafast universal RNA-seq aligner. Bioinformatics 29, 15-21.

Farabaugh, P.J., Zhao, H., and Vimaladithan, A. (1993). A novel programed frameshift expresses the POL3 gene of retrotransposon Ty3 of yeast: frameshifting without tRNA slippage. Cell 74, 93-103.

Feng, Y.X., Moore, S.P., Garfinkel, D.J., and Rein, A. (2000). The genomic RNA in Ty1 virus-like particles is dimeric. J. Virol. 74, 10819-10821.

Frankish, A., Diekhans, M., Ferreira, A.M., Johnson, R., Jungreis, I., Loveland, J., Mudge, J.M., Sisu, C., Wright, J., Armstrong, J., et al. (2019). GENCODE reference annotation for the human and mouse genomes. Nucleic Acids Res. 47, D766-D773.

Garfinkel, D.J., Boeke, J.D., and Fink, G.R. (1985). Ty element transposition: reverse transcriptase and virus-like particles. Cell 42, 507-517.

Griffith, J.L., Coleman, L.E., Raymond, A.S., Goodson, S.G., Pittard, W.S., Tsui, C., and Devine, S.E. (2003). Functional genomics reveals relationships between the retrovirus-like Ty1 element and its host Saccharomyces cerevisiae. Genetics 164, 867-879.

Haeussler, M., Zweig, A.S., Tyner, C., Speir, M.L., Rosenbloom, K.R., Raney, B.J., Lee, C.M., Lee, B.T., Hinrichs, A.S., Gonzalez, J.N., et al. (2019). The UCSC genome browser database: 2019 update. Nucleic Acids Res. 47, D853-D858.

Hahne, F., and Ivanek, R. (2016). Visualizing genomic data using Giviz and bioconductor. Methods Mol. Biol. 1418, 335-351.

Haig, D. (2016). Transposable elements: self-seekers of the germline, team-players of the soma. BioEssays 38, 1158-1166.

Hayward, A. (2017). Origin of the retroviruses: when, where, and how? Curr. Opin. Virol. 25, 23-27.

Irwin, B., Aye, M., Baldi, P., Beliakova-Bethell, N., Cheng, H., Dou, Y., Liou, W., and Sandmeyer, S. (2005). Retroviruses and yeast retrotransposons use overlapping sets of host genes. Genome Res. 15, 641-654.

Isono, E., Saito, N., Kamata, N., Saeki, Y., and Toh-E, A. (2005). Functional analysis of Rpn6p, a lid component of the $26 \mathrm{~S}$ proteasome, using temperature-sensitive rpn6 mutants of the yeast Saccharomyces cerevisiae. J. Biol. Chem. 280, 6537-6547.

Jin, L., Zhang, K., Sternglanz, R., and Neiman, A.M. (2017). Predicted RNA binding proteins Pes4 and Mip6 regulate mRNA levels, translation, and localization during sporulation in budding yeast. Mol. Cell. Biol. 37, 10-12.

Kane, S.M., and Roth, R. (1974). Carbohydrate metabolism during ascospore development in yeast. J. Bacteriol. 118, 8-14.

Kano, H., Godoy, I., Courtney, C., Vetter, M.R., Gerton, G.L., Ostertag, E.M., and Kazazian, H.H. (2009). L1 retrotransposition occurs mainly in embryogen esis and creates somatic mosaicism. Genes Dev. 23, 1303-1312.

Kim, D., Pertea, G., Trapnell, C., Pimentel, H., Kelley, R., and Salzberg, S.L. (2013). TopHat2: accurate alignment of transcriptomes in the presence of insertions, deletions and gene fusions. Genome Biol. 14, R36.

King, O.D., Gitler, A.D., and Shorter, J. (2012). The tip of the iceberg: RNAbinding proteins with prion-like domains in neurodegenerative disease. Brain Res. 1462, 61-80.

Klutstein, M., Siegfried, Z., Gispan, A., Farkash-Amar, S., Zinman, G., BarJoseph, Z., Simchen, G., and Simon, I. (2010). Combination of genomic approaches with functional genetic experiments reveals two modes of repression of yeast middle-phase meiosis genes. BMC Genomics 11, 478.

Kojima, K.K. (2018). Human transposable elements in Repbase: genomic footprints from fish to humans. Mob. DNA 9, 2. 


\section{CellPress}

OPEN ACCESS

Developmental Cell

Article

Kojima, M.L., de Rooij, D.G., and Page, D.C. (2019). Amplification of a broad transcriptional program by a common factor triggers the meiotic cell cycle in mice. eLife 8, e43738.

Kokošar, J., and Kordiš, D. (2013). Genesis and regulatory wiring of retroelement-derived domesticated genes: a phylogenomic perspective. Mol. Biol. Evol. 30, 1015-1031.

Kronja, I., and Orr-Weaver, T.L. (2011). Translational regulation of the cell cycle: when, where, how and why? Philos. Trans. R. Soc. Lond. B Biol. Sci. 366, 3638-3652.

Langmead, B., and Salzberg, S.L. (2012). Fast gapped-read alignment with Bowtie 2. Nat. Methods 9, 357-359.

Larsen, L.S.Z., Beliakova-Bethell, N., Bilanchone, V., Zhang, M., Lamsa, A., DaSilva, R., Hatfield, G.W., Nagashima, K., and Sandmeyer, S. (2008). Ty3 nucleocapsid controls localization of particle assembly. J. Virol. 82, 2501-2514.

Lee, B., and Amon, A. (2001). Meiosis: how to create a specialized cell cycle. Curr. Opin. Cell Biol. 13, 770-777.

Lesch, B.J., Silber, S.J., McCarrey, J.R., and Page, D.C. (2016). Parallel evoIution of male germline epigenetic poising and somatic development in animals. Nat. Genet. 48, 888-894.

Li, H., and Durbin, R. (2009). Fast and accurate short read alignment with Burrows-Wheeler transform. Bioinformatics 25, 1754-1760.

Li, H., Handsaker, B., Wysoker, A., Fennell, T., Ruan, J., Homer, N., Marth, G., Abecasis, G., and Durbin, R.; 1000 Genome Project Data Processing Subgroup (2009). The sequence alignment/map format and SAMtools. Bioinformatics 25, 2078-2079.

Longtine, M.S., Mckenzie, A., III, Demarini, D.J., Shah, N.G., Wach, A., Brachat, A., Philippsen, P., and Pringle, J.R. (1998). Additional modules for versatile and economical PCR-based gene deletion and modification in Saccharomyces cerevisiae. Yeast 14, 953-961.

MacLennan, M., García-Cañadas, M., Reichmann, J., Khazina, E., Wagner, G., Playfoot, C.J., Salvador-Palomeque, C., Mann, A.R., Peressini, P., Sanchez, L., et al. (2017). Mobilization of LINE-1 retrotransposons is restricted by Tex19. 1 In mouse embryonic stem cells. eLife 6, e26152.

Malki, S., van der Heijden, G.W., O'Donnell, K.A., Martin, S.L., and Bortvin, A. (2014). A role for retrotransposon LINE-1 in fetal oocyte attrition in mice. Dev. Cell 29, 521-533.

Marin, R., Cortez, D., Lamanna, F., Pradeepa, M.M., Leushkin, E., Julien, P., Liechti, A., Halbert, J., Brüning, T., Mössinger, K., et al. (2017). Convergent origination of a Drosophila-like dosage compensation mechanism in a reptile lineage. Genome Res. 27, 1974-1987.

Martin, M. (2011). Cutadapt removes adapter sequences from highthroughput sequencing reads. EMBnet J. 17, 10-12.

Maxwell, P.H., and Curcio, M.J. (2007). Host factors that control long terminal repeat retrotransposons in Saccharomyces cerevisiae: implications for regulation of mammalian retroviruses. Eukaryot. Cell 6, 1069-1080.

Merhej, J., Frigo, A., Le Crom, S., Camadro, J.M., Devaux, F., and Lelandais, G. (2014). bPeaks: a bioinformatics tool to detect transcription factor binding sites from ChlPseq data in yeasts and other organisms with small genomes. Yeast 31, 375-391.

Mita, P., and Boeke, J.D. (2016). How retrotransposons shape genome regulation. Curr. Opin. Genet. Dev. 37, 90-100.

Morawska, M., and Ulrich, H.D. (2013). An expanded tool kit for the auxin-inducible degron system in budding yeast. Yeast 30, 341-351.

Nocedal, I., Mancera, E., and Johnson, A.D. (2017). Gene regulatory network plasticity predates a switch in function of a conserved transcription regulator. eLife 6, 1-20.

Nymark-McMahon, M.H., Beliakova-Bethell, N.S., Darlix, J.L., Le Grice, S.F., and Sandmeyer, S.B. (2002). Ty3 integrase is required for initiation of reverse transcription. J. Virol. 76, 2804-2816.

Orecchini, E., Frassinelli, L., Galardi, S., Ciafrè, S.A., and Michienzi, A. (2018). Post-transcriptional regulation of LINE-1 retrotransposition by AID/APOBEC and ADAR deaminases. Chromosome Res. 26, 45-59.
Peter, J., De Chiara, M., Friedrich, A., Yue, J.-X., Pflieger, D., Bergström, A., Sigwalt, A., Barre, B., Freel, K., Llored, A., et al. (2018). Genome evolution across 1,011 Saccharomyces cerevisiae isolates. Nature 556, 339-344.

Pierce, M., Benjamin, K.R., Montano, S.P., Georgiadis, M.M., Winter, E., and Vershon, A.K. (2003). Sum1 and Ndt80 proteins compete for binding to middle sporulation element sequences that control meiotic gene expression. Mol. Cell. Biol. 23, 4814-4825.

Qi, X., Daily, K., Nguyen, K., Wang, H., Mayhew, D., Rigor, P., Forouzan, S., Johnston, M., Mitra, R.D., Baldi, P., and Sandmeyer, S. (2012). Retrotransposon profiling of RNA polymerase III initiation sites. Genome Res. 22, 681-692.

Quinlan, A.R., and Hall, I.M. (2010). BEDTools: a flexible suite of utilities for comparing genomic features. Bioinformatics 26, 841-842.

Ramírez, F., Ryan, D.P., Grüning, B., Bhardwaj, V., Kilpert, F., Richter, A.S., Heyne, S., Dündar, F., and Manke, T. (2016). deepTools2: a next generation web server for deep-sequencing data analysis. Nucleic Acids Res. 44, W160-W165.

Rebollo, R., Romanish, M.T., and Mager, D.L. (2012). Transposable elements: an abundant and natural source of regulatory sequences for host genes. Annu. Rev. Genet. 46, 21-42.

Ribeiro-dos-Santos, G., Schenberg, A.C.G., Gardner, D.C.J., and Oliver, S.G. (1997). Enhancement of Ty transposition at the ADH4 and ADH2 loci in meiotic yeast cells. Mol. Gen. Genet. 254, 555-561.

Robinson, M.D., McCarthy, D.J., and Smyth, G.K. (2010). edgeR: a Bioconductor package for differential expression analysis of digital gene expression data. Bioinformatics 26, 139-140.

Rosario, R., Smith, R.W.P., Adams, I.R., and Anderson, R.A. (2017). RNA immunoprecipitation identifies novel targets of DAZL in human foetal ovary. Mol. Hum. Reprod. 23, 177-186.

Rowley, P.A., Patterson, K., Sandmeyer, S.B., and Sawyer, S.L. (2018). Control of yeast retrotransposons mediated through nucleoporin evolution. PLoS Genet. 14, e1007325.

Sawyer, E.M., Joshi, P.R., Jorgensen, V., Yunus, J., Berchowitz, L.E., and Ünal, E. (2019). Developmental regulation of an organelle tether coordinates mitochondrial remodeling in meiosis. J. Cell Biol. 218, 559-579.

Schindelin, J., Arganda-Carreras, I., Frise, E., Kaynig, V., Longair, M., Pietzsch, T., Preibisch, S., Rueden, C., Saalfeld, S., Schmid, B., et al. (2012). Fiji: an open-source platform for biological-image analysis. Nat. Methods 9, 676-682.

Scholes, D.T., Banerjee, M., Bowen, B., and Curcio, M.J. (2001). Multiple regulators of Ty1 transposition in Saccharomyces cerevisiae have conserved roles in genome maintenance. Genetics 159, 1449-1465.

Servant, G., Pennetier, C., and Lesage, P. (2008). Remodeling yeast gene transcription by activating the Ty 1 long terminal repeat retrotransposon under severe adenine deficiency. Mol. Cell. Biol. 28, 5543-5554.

Shen, X.-X., Opulente, D.A., Kominek, J., Zhou, X., Steenwyk, J.L., Buh, K.V., Haase, M.A.B., Wisecaver, J.H., Wang, M., Doering, D.T., et al. (2018). Tempo and mode of genome evolution in the budding yeast subphylum. Cell 175, 1533-1545.e20.

Soumillon, M., Necsulea, A., Weier, M., Brawand, D., Zhang, X., Gu, H., Barthès, P., Kokkinaki, M., Nef, S., Gnirke, A., et al. (2013). Cellular source and mechanisms of high transcriptome complexity in the mammalian testis. Cell Rep. 3, 2179-2190.

Storici, F., Lewis, L.K., and Resnick, M.A. (2001). In vivo site-directed mutagenesis using oligonucleotides. Nat. Biotechnol. 19, 773-776.

Thorvaldsdóttir, H., Robinson, J.T., and Mesirov, J.P. (2013). Integrative Genomics Viewer (IGV): high-performance genomics data visualization and exploration. Brief. Bioinform. 14, 178-192.

Torres, E.M., Sokolsky, T., Tucker, C.M., Chan, L.Y., Boselli, M., Dunham, M.J., and Amon, A. (2007). Effects of aneuploidy on cellular physiology and cell division in haploid yeast. Science 317, 916-924.

Tsuchiya, D., Yang, Y., and Lacefield, S. (2014). Positive feedback of NDT80 expression ensures irreversible meiotic commitment in budding yeast. PLoS Genet. 10, e1004398. 
Volff, J.N. (2006). Turning junk into gold: domestication of transposable elements and the creation of new genes in eukaryotes. BioEssays 28, 913-922.

Volff, J.N. (2009). Cellular genes derived from Gypsy/Ty3 retrotransposons in mammalian genomes. Ann. N Y Acad. Sci. 1178, 233-243.

Wang, L., Dou, K., Moon, S., Tan, F.J., and Zhang, Z.Z.Z. (2018). Hijacking oogenesis enables massive propagation of LINE and retroviral transposons. Cell 174, 1082-1094.e12.

Warren, I.A., Naville, M., Chalopin, D., Levin, P., Berger, C.S., Galiana, D., and Volff, J.N. (2015). Evolutionary impact of transposable elements on genomic diversity and lineage-specific innovation in vertebrates. Chromosome Res. 23, 505-531.

Wicker, T., Sabot, F., Hua-Van, A., Bennetzen, J.L., Capy, P., Chalhoub, B., Flavell, A., Leroy, P., Morgante, M., Panaud, O., et al. (2007). A unified classification system for eukaryotic transposable elements. Nat. Rev. Genet. 8, 973-982.

Winter, E. (2012). The Sum1/Ndt80 transcriptional switch and commitment to meiosis in Saccharomyces cerevisiae. Microbiol. Mol. Biol. Rev. 76, 1-15.
Xu, L., Ajimura, M., Padmore, R., Klein, C., and Kleckner, N. (1995). NDT80, a meiosis-specific gene required for exit from pachytene in Saccharomyces cerevisiae. Mol. Cell. Biol. 15, 6572-6581.

Yang, F., and Wang, P.J. (2016). Multiple LINEs of retrotransposon silencing mechanisms in the mammalian germline. Semin. Cell Dev. Biol. 59, 118-125.

Yue, J.-X.X., Li, J., Aigrain, L., Hallin, J., Persson, K., Oliver, K., Bergström, A., Coupland, P., Warringer, J., Lagomarsino, M.C., et al. (2017). Contrasting evolutionary genome dynamics between domesticated and wild yeasts. Nat. Genet. 49, 913-924.

Zagore, L.L., Sweet, T.J., Hannigan, M.M., Weyn-Vanhentenryck, S.M., Jobava, R., Hatzoglou, M., Zhang, C., and Licatalosi, D.D. (2018). DAZL regulates germ cell survival through a network of polyA-proximal mRNA interactions. Cell Rep. 25, 1225-1240.e6.

Zhang, Y., Liu, T., Meyer, C.A., Eeckhoute, J., Johnson, D.S., Bernstein, B.E., Nusbaum, C., Myers, R.M., Brown, M., Li, W., and Liu, S. (2008). Model-based analysis of ChIP-seq (MACS). Genome Biol. 9, R137.

Zhou, L., Canagarajah, B., Zhao, Y., Baibakov, B., Tokuhiro, K., Maric, D., and Dean, J. (2017). BTBD18 regulates a subset of piRNA-generating loci through transcription elongation in mice. Dev. Cell 40, 453-466.e5. 


\section{STAR $\star$ METHODS}

\section{KEY RESOURCES TABLE}

\begin{tabular}{|c|c|c|}
\hline REAGENT or RESOURCE & SOURCE & IDENTIFIER \\
\hline \multicolumn{3}{|l|}{ Antibodies } \\
\hline$\alpha-\mathrm{HA} .11$ Clone:16B12 (mouse) & BioLegend & Cat\# 901514 \\
\hline $\begin{array}{l}\alpha \text {-Pgk1 (Phosphoglycerate Kinase } \\
\text { monoclonal) (mouse) }\end{array}$ & Novex (Life Technologies) & Part\# 459250 \\
\hline$\alpha$-v5 monoclonal (mouse) & Invitrogen & Cat\# 46-0705 \\
\hline$\alpha$-FLAG (rabbit) & Sigma-Aldrich & Cat\# F7425 \\
\hline$\alpha$-VLP (Ty3) crude serum (rabbit) & Sandmeyer lab (UC Irvine) & $\alpha$-VLP (Ty3) crude serum \\
\hline $\begin{array}{l}\alpha \text {-VLP (Ty3) affinity purified antibody, using } \\
\text { E. coli produced Gag3 protein }\end{array}$ & This study & $\alpha-\operatorname{VLP}(\mathrm{Ty} 3)$ affinity purified antibody \\
\hline$\alpha$-mouse HRP-conjugated secondary & GE Healthcare & Cat\# NA931-1ML \\
\hline$\alpha$-rabbit HRP-conjugated secondary & GE Healthcare & Cat\# NA934V \\
\hline$\alpha$-V5-coupled agarose & Sigma-Aldrich & Cat\# A7345-1ml \\
\hline Rat $\alpha$-tubulin alpha & Bio-Rad & Cat\# MCA77G \\
\hline$\alpha$-rat-FITC & Invitrogen & Cat\# 31629 \\
\hline \multicolumn{3}{|l|}{ Chemicals, Peptides, and Recombinant Proteins } \\
\hline Halt Protease Inhibitor & ThermoFisher & Cat\# 1861279 \\
\hline ProlongGold anti-fade reagent w/ DAPI & Invitrogen & Cat\# P36935 \\
\hline$\beta$-Estradiol & Sigma-Aldrich & Cat\# E8875 \\
\hline MG-132 & Cayman Chemical & Cat\# 10012628 \\
\hline Auxin (indole-3-acetic acid) & Sigma-Aldrich & Cat\# 12886-25G \\
\hline$\alpha$-factor mating pheromone & Genscript & Cat\# RP01002 \\
\hline Acid-washed glass beads, $425-600 \mu \mathrm{m}$ & Sigma-Aldrich & Cat\# G8772-500G \\
\hline $0.5 \mathrm{~mm}$ dia Zirconia/silica beads & BioSpec Products & Cat\# $11079105 Z$ \\
\hline Chelex resin & Bio-Rad & Cat\# 1422832 \\
\hline Hybond-N+ membrane & GE Healthcare & Prod\# RPN203B \\
\hline $\begin{array}{l}\text { 10\% Criterion TGX Precast Midi Protein } \\
\text { Gel, } 26 \text { well, } 15 \mu \mathrm{l}\end{array}$ & Bio-Rad & Cat\# 5671035 \\
\hline $\begin{array}{l}\text { Trans-blot turbo transfer kits } \\
\text { (Nitrocellulose) }\end{array}$ & Bio-Rad & Cat\# 1704271 \\
\hline Illustra Probequant Columns & GE Healthcare & Prod\# 28903408 \\
\hline \multicolumn{3}{|l|}{ Critical Commercial Assays } \\
\hline AminoLink Plus Immobilization kit & Thermo Scientific & Cat\# 44894 \\
\hline Amersham MegaPrime DNA Labeling Kit & GE Healthcare & Prod\# RPN1604 \\
\hline Minelute kit & Qiagen & Cat\# 28004 \\
\hline Accel-NGS 1S plus DNA library kit & Swift Biosciences & Cat\# 10096 \\
\hline TruSeq Stranded Total Rna Kit & Illumina & Cat\# RS-122-2302 \\
\hline \multicolumn{3}{|l|}{ Deposited data } \\
\hline $\begin{array}{l}\text { SK1 reference genome and other } \\
\text { S. cerevisiae and S. paradoxus reference } \\
\text { genomes }\end{array}$ & (Yue et al., 2017) & $\begin{array}{l}\text { https://yjx1217.github.io/ } \\
\text { Yeast_PacBio_2016/data/ }\end{array}$ \\
\hline $\begin{array}{l}\text { Raw reads for RNA-seq and ChIP-seq EBI } \\
\text { ENA / NCBI BioProject PRJNA669383 }\end{array}$ & This study & $\begin{array}{l}\text { https://www.ncbi.nlm.nih.gov/bioproject/? } \\
\text { term=PRJNA669383 }\end{array}$ \\
\hline Mouse reference genome: $\mathrm{mm} 10$ assembly & Mouse Genome Sequencing Consortium & https://genome.ucsc.edu/ \\
\hline $\begin{array}{l}\text { RNAseq adult mouse testicular cell types, } \\
\text { EBI ENA / NCBI BioProject: PRJNA187158 }\end{array}$ & (Soumillon et al., 2013) & $\begin{array}{l}\text { https://www.ebi.ac.uk/ena/browser/view/ } \\
\text { PRJNA187158 }\end{array}$ \\
\hline
\end{tabular}




\begin{tabular}{|c|c|c|}
\hline Continued & & \\
\hline REAGENT or RESOURCE & SOURCE & IDENTIFIER \\
\hline $\begin{array}{l}\text { RNAseq for P14 Myb/1 testes EBI ENA / } \\
\text { NCBI BIOPROJECT: PRJNA321732 }\end{array}$ & (Zhou et al., 2017) & $\begin{array}{l}\text { https://www.ebi.ac.uk/ena/browser/view/ } \\
\text { PRJNA321732 }\end{array}$ \\
\hline $\begin{array}{l}\text { RNAseq Stra8 preleptotene spermatocytes } \\
\text { EBI ENA / NCBI BIOPROJECT: } \\
\text { PRJNA476515 }\end{array}$ & (Kojima et al., 2019) & $\begin{array}{l}\text { https://www.ebi.ac.uk/ena/browser/view/ } \\
\text { PRJNA476515 }\end{array}$ \\
\hline Repeatmasker-annotated loci in mm10 & Institute for Systems Biology & http://repeatmasker.org/species/mm.html \\
\hline $\begin{array}{l}\text { GencodeBasic VM18 annotation NCBI } \\
\text { GEO: GSE108181 }\end{array}$ & GENCODE project & $\begin{array}{l}\text { https://www.gencodegenes.org/mouse/ } \\
\text { release_M18.html }\end{array}$ \\
\hline $\begin{array}{l}\text { Mybl1 ChIPseq data from mouse testes EBI } \\
\text { ENA / NCBI BIOPROJECT: PRJNA321732 }\end{array}$ & (Kojima et al., 2019) & $\begin{array}{l}\text { https://www.ebi.ac.uk/ena/browser/view/ } \\
\text { PRJNA321732 }\end{array}$ \\
\hline $\begin{array}{l}\text { Stra8 ChIPseq data from mouse testes EBI } \\
\text { ENA / NCBI BIOPROJECT: PRJNA476515 }\end{array}$ & (Kojima et al., 2019) & $\begin{array}{l}\text { https://www.ebi.ac.uk/ena/browser/view/ } \\
\text { PRJNA476515 }\end{array}$ \\
\hline $\begin{array}{l}\text { Dazl CLIP from mouse testes NCBI GEO: } \\
\text { GSE108181 }\end{array}$ & (Zagore et al., 2018) & $\begin{array}{l}\text { https://www.ncbi.nlm.nih.gov/geo/query/ } \\
\text { acc.cgi?acc=GSE108181 }\end{array}$ \\
\hline $\begin{array}{l}\text { RNA-seq for opossum (purified } \\
\text { spermatocyte/spermatid) NCBI GEO: } \\
\text { GSE68507 }\end{array}$ & (Lesch et al., 2016) & $\begin{array}{l}\text { https://www.ncbi.nlm.nih.gov/geo/query/ } \\
\text { acc.cgi?acc=GSE68507 }\end{array}$ \\
\hline $\begin{array}{l}\text { RNA-seq from opossum (multitissue) NCBI } \\
\text { GEO: GSE97367 }\end{array}$ & (Marin et al., 2017) & $\begin{array}{l}\text { https://www.ncbi.nlm.nih.gov/geo/query/ } \\
\text { acc.cgi?acc=GSE97367 }\end{array}$ \\
\hline Human reference genome: hg38 assembly & Genome Reference Consortium & https://genome.ucsc.edu/ \\
\hline Repeatmasker-annotated loci in hg38 & Institute for Systems Biology & http://repeatmasker.org/species/hg.html \\
\hline $\begin{array}{l}\text { DAZL RIP from human ovaries EBI ENA / } \\
\text { NCBI BIOPROJECT: PRJNA321858 }\end{array}$ & (Rosario et al., 2017) & $\begin{array}{l}\text { https://www.ebi.ac.uk/ena/browser/view/ } \\
\text { PRJNA321858 }\end{array}$ \\
\hline \multicolumn{3}{|l|}{ Experimental Models: Organisms/Strains } \\
\hline $\begin{array}{l}\text { All lab strains are S. cerevisiae, of SK1 } \\
\text { background (see Table S1) }\end{array}$ & (Kane and Roth, 1974) & ATCC: 204722 \\
\hline \multicolumn{3}{|l|}{ Oligonucleotides } \\
\hline $\begin{array}{l}\text { FW primer for Ty3 Northern probe: } \\
\text { 5'-GCTTTATGGATCAAATCCCC-3' }\end{array}$ & This paper & N/A \\
\hline $\begin{array}{l}\text { RV primer for Ty3 Northern probe: } \\
\text { 5'-AGCATATATCGGAAGTGGTGGA-3' }\end{array}$ & This paper & $\mathrm{N} / \mathrm{A}$ \\
\hline $\begin{array}{l}\text { FW primer for CA Northern probe: } \\
\text { 5'-GCTTTATGGATCAAATCCCC-3' }\end{array}$ & This paper & N/A \\
\hline $\begin{array}{l}\text { RV primer for CA Northern probe: } \\
\text { 5'-ACCGATGATAGTGTCTCC-3' }\end{array}$ & This paper & N/A \\
\hline $\begin{array}{l}\text { FW primer for PR Northern probe: } \\
\text { 5'-TATATCGCCATCCCCGAGATGG-3' }\end{array}$ & This paper & $\mathrm{N} / \mathrm{A}$ \\
\hline $\begin{array}{l}\text { RV primer for PR Northern probe: } \\
\text { 5'-GACAACATTGGAGTATTTTCC-3' }\end{array}$ & This paper & N/A \\
\hline $\begin{array}{l}\text { FW primer for RT Northern probe: } \\
\text { 5'-GATAACAAGTTCATTGTTCCC-3' }\end{array}$ & This paper & $\mathrm{N} / \mathrm{A}$ \\
\hline $\begin{array}{l}\text { RV primer for RT Northern probe: } \\
\text { 5'-GGAGCAATTTGGAATGAATCG-3' }\end{array}$ & This paper & $\mathrm{N} / \mathrm{A}$ \\
\hline $\begin{array}{l}\text { FW primer for IN Northern probe: } \\
\text { 5'-GACGCCTCAAAAGACGGC-3' }\end{array}$ & This paper & $\mathrm{N} / \mathrm{A}$ \\
\hline $\begin{array}{l}\text { RV primer for IN Northern probe: } \\
\text { 5'-TTCCAAGTGTTCTAGTAGG-3' }\end{array}$ & This paper & N/A \\
\hline \multicolumn{3}{|l|}{ Recombinant DNA } \\
\hline $\begin{array}{l}\text { Construct: ura3::pGPD- } \\
\text { GAL4(848).ER::URA3 }\end{array}$ & (Benjamin et al., 2003) & N/A \\
\hline Construct: pGAL-NDT80::TRP1 & (Benjamin et al., 2003) & N/A \\
\hline Construct: CLB3-3HA:kanMX6 & (Carlile and Amon, 2008) & N/A \\
\hline Construct: RIM4-3V5::His3MX6 & (Berchowitz et al., 2013) & $\mathrm{N} / \mathrm{A}$ \\
\hline
\end{tabular}




\begin{tabular}{|c|c|c|}
\hline \multicolumn{3}{|l|}{ Continued } \\
\hline REAGENT or RESOURCE & SOURCE & IDENTIFIER \\
\hline Construct: RIM4-AID*6FLAG::HYGR & this paper & $\mathrm{N} / \mathrm{A}$ \\
\hline Construct: rim4 $4138-3 \mathrm{~V} 5:: \mathrm{His} 3 \mathrm{MX} 6$ & (Berchowitz et al., 2015) & $\mathrm{N} / \mathrm{A}$ \\
\hline Construct: rim4 $4271-3 \mathrm{~V} 5:: \mathrm{His} 3 \mathrm{MX} 6$ & (Berchowitz et al., 2015) & $\mathrm{N} / \mathrm{A}$ \\
\hline Construct: pdr5::TRP1 & (Torres et al., 2007) & $\mathrm{N} / \mathrm{A}$ \\
\hline Construct: rpn6-1::His3MX6 & (Carpenter et al., 2018) & $\mathrm{N} / \mathrm{A}$ \\
\hline Construct: ty $3 \Delta$ & this paper & $\mathrm{N} / \mathrm{A}$ \\
\hline Construct: MSE-His3MX6-Ty3 & this paper & $\mathrm{N} / \mathrm{A}$ \\
\hline Construct: kanMX6-Ty3 & this paper & $\mathrm{N} / \mathrm{A}$ \\
\hline $\begin{array}{l}\text { Construct: kanMX6-mse(-269) } \\
\triangle:: A A A A A A-+T T(-163)-m s e(-79) \\
\Delta:: A A A A A A-T y 3\end{array}$ & this paper & $\mathrm{N} / \mathrm{A}$ \\
\hline Construct: his3::pGPD1-OsTIR1::His3MX6 & this paper & $\mathrm{N} / \mathrm{A}$ \\
\hline Construct: his3::pRIM4-OsTIR1::His3MX6 & this paper & $\mathrm{N} / \mathrm{A}$ \\
\hline Construct: SUM1-3V5-IAA7-kanMX & this paper & $\mathrm{N} / \mathrm{A}$ \\
\hline $\begin{array}{l}\text { Plasmid: pHyg-AID*-6FLAG (to build RIM4- } \\
\text { AID*-6FLAG::HYGR) }\end{array}$ & (Morawska and Ulrich, 2013) & https://www.addgene.org/99519/ \\
\hline $\begin{array}{l}\text { Plasmid: pFA6a-kanMX6 (to build kanMX6- } \\
\text { Ty3 and kanMX6-mse(-269) } \triangle:: A A A A A A- \\
T T(-163)-m s e(-79) \triangle:: A A A A A A-T y 3))\end{array}$ & (Longtine et al., 1998) & https://www.addgene.org/39296/ \\
\hline $\begin{array}{l}\text { Plasmid: pLZL2422 (to produce Gag3 } \\
\text { protein to purify } \alpha \text {-VLP from crude serum) }\end{array}$ & (Larsen et al., 2008) & $\mathrm{N} / \mathrm{A}$ \\
\hline $\begin{array}{l}\text { Plasmid: pFA6a-His3MX6 (to build MSE- } \\
\text { His3MX6-Ty3) }\end{array}$ & (Longtine et al., 1998) & https://www.addgene.org/41596/ \\
\hline $\begin{array}{l}\text { Plasmid: pUB976 (to make his3::pRIM4- } \\
\text { OsTIR1::His3MX6 and his3:::pGPD1- } \\
\text { OsTIR1::His3MX6) }\end{array}$ & (Sawyer et al., 2019) & $\mathrm{N} / \mathrm{A}$ \\
\hline \multicolumn{3}{|l|}{ Software and Algorithms } \\
\hline R v3.6.1 & R Core Team & https://www.R-project.org/ \\
\hline R studio v1.1.383 & R Core Team & http://www.rstudio.com/ \\
\hline STAR v020201 & (Dobin et al., 2013) & https://github.com/alexdobin/STAR \\
\hline Ving software $v$ beta1.1 & (Descrimes et al., 2015) & http://vm-gb.curie.fr/ving/ \\
\hline cutadapt v2.10 & (Martin, 2011) & $\begin{array}{l}\text { https://cutadapt.readthedocs.io/en/stable/ } \\
\text { index.html }\end{array}$ \\
\hline $\begin{array}{l}\text { Bowtie2 v2.3.4 (yeast analysis) and v2.2.6 } \\
\text { (mammals analysis) }\end{array}$ & (Langmead and Salzberg, 2012) & $\begin{array}{l}\text { https://anaconda.org/bioconda/ } \\
\text { bowtie2/files }\end{array}$ \\
\hline SAMtools v1.6 & (Li and Durbin, 2009) & http://www.htslib.org/ \\
\hline $\begin{array}{l}\text { Picard tool MarkDuplicates v } 2.23 .3 \text { (yeast } \\
\text { analysis) and v2.17.11 (mammals analysis) }\end{array}$ & Broad Institute & https://broadinstitute.github.io/picard/ \\
\hline Deeptools bamcoverage & (Ramírez et al., 2016) & $\begin{array}{l}\text { https://deeptools.readthedocs.io/en/ } \\
\text { develop/content/tools/bamCoverage.html }\end{array}$ \\
\hline Yeast ChIP normalization custom script & Available upon request & $\mathrm{N} / \mathrm{A}$ \\
\hline bpeaks v1.2 & (Merhej et al., 2014) & $\begin{array}{l}\text { https://cran.r-project.org/web/packages/ } \\
\text { bPeaks/index.html }\end{array}$ \\
\hline IGV v2.8.9 & (Thorvaldsdóttir et al., 2013) & $\begin{array}{l}\text { http://software.broadinstitute.org/ } \\
\text { software/igv/ }\end{array}$ \\
\hline Fiji software & (Schindelin et al., 2012) & https://fiji.sc/ \\
\hline TrimGalore 0.4.1 & The Babraham Institute & $\begin{array}{l}\text { https://github.com/FelixKrueger/ } \\
\text { TrimGalore }\end{array}$ \\
\hline Tophat v2.1.0 & (Kim et al., 2013) & $\begin{array}{l}\text { https://ccb.jhu.edu/software/tophat/ } \\
\text { index.shtml }\end{array}$ \\
\hline EdgeR & (Robinson et al., 2010) & $\begin{array}{l}\text { http://bioconductor.org/packages/release/ } \\
\text { bioc/html/edgeR.html }\end{array}$ \\
\hline
\end{tabular}




\begin{tabular}{|c|c|c|}
\hline \multicolumn{3}{|l|}{ Continued } \\
\hline REAGENT or RESOURCE & SOURCE & IDENTIFIER \\
\hline Htseq-count v0.11.2 & (Anders et al., 2015) & $\begin{array}{l}\text { https://htseq.readthedocs.io/en/release_0. } \\
\text { 11.1/count.html }\end{array}$ \\
\hline bedtools v2.26.0 & (Quinlan and Hall, 2010) & $\begin{array}{l}\text { https://github.com/arq5x/bedtools2/ } \\
\text { releases/tag/v2.26.0 }\end{array}$ \\
\hline MACS v2.1.1 & (Zhang et al., 2008) & https://github.com/macs3-project/MACS \\
\hline Gviz & (Hahne and Ivanek, 2016) & $\begin{array}{l}\text { http://bioconductor.org/packages/release/ } \\
\text { bioc/html/Gviz.html }\end{array}$ \\
\hline UCSC liftOver tool & (Haeussler et al., 2019) & $\begin{array}{l}\text { https://genome.ucsc.edu/cgi-bin/ } \\
\text { hgLiftOver }\end{array}$ \\
\hline \multicolumn{3}{|l|}{ Other } \\
\hline DeltaVision Elite Microcope & GE Healthcare & Prod \#: 29065728 \\
\hline FastPrep-24 & MP biomedicals & Cat\# 6004500 \\
\hline Criterion Vertical Electrophoresis Cell & Bio-Rad & Cat\# 1656001 \\
\hline Trans-Blot Turbo Transfer System & Bio-Rad & Cat\# 1704150 \\
\hline
\end{tabular}

\section{RESOURCE AVAILABILITY}

\section{Lead Contact}

Further information and requests for resources and reagents should be directed to and will be fulfilled by the Lead Contact, Luke E. Berchowitz (leb2210@cumc.columbia.edu).

\section{Materials Availability}

All strains and reagents used in this study are available upon request.

Data and Code Availability

The accession number for the RNA sequencing and ChIP sequencing dataset generated during this study is EBI ENA / NCBI BioProject: PRJNA669383 (https://www.ncbi.nlm.nih.gov/bioproject/?term=PRJNA669383; https://www.ebi.ac.uk/ena/browser/view/ PRJNA669383).

Custom code and algorithms are available upon request.

\section{EXPERIMENTAL MODEL AND SUBJECT DETAILS}

\section{Yeast Strains and Constructs}

Lab strains are described in Table S1, and wild strains in Table S2. Strains were constructed by crossing and/or using the PCR-based method described in (Longtine et al., 1998) using the SK1 genome reference and annotation from (Yue et al., 2017). PCR and fusion PCR involved templates pFA6a-kanMX6 and pFA6a-His3MX6 (Longtine et al., 1998) and pUB976 (Sawyer et al., 2019). Seamless deletion of Ty3 was introduced by pop-in pop-out procedure as described in (Storici et al., 2001). The origins of all constructs are listed in the Key Resources Table (this study, or (Benjamin et al., 2003; Berchowitz et al., 2013, 2015; Carlile and Amon, 2008; Carpenter et al., 2018; Torres et al., 2007)).

\section{METHOD DETAILS}

\section{Yeast Growth and Sporulation Conditions}

All yeast strains were grown at $30^{\circ} \mathrm{C}$. For sporulation of lab strains, strains were pulled from $-80^{\circ} \mathrm{C}$ stock and grown on YPG (3\% glycerol) plates overnight, were then transferred to YPD 4\% (YPD + 4\% glucose) plates for $24 \mathrm{~h}$, were then grown to saturation in YPD for 24 h, diluted in BYTA ( $1 \%$ yeast extract, $2 \%$ tryptone, $1 \%$ potassium acetate, $50 \mathrm{mM}$ potassium pthalate) to $\mathrm{OD}_{600}=0.3$ and grown overnight. Cells were washed with water, resuspended in sporulation medium $(0.3 \%$ potassium acetate $[\mathrm{pH} 7.0], 0.02 \%$ raffinose $)$ to $\mathrm{OD}_{600}=$ 1.8. Following inoculation into sporulation medium, cells were incubated with vigorous shaking $(\mathrm{t}=0 \mathrm{~h})$. For Rim4-deg strain, at $\mathrm{t}=5 \mathrm{~h}$, auxin (indole-3-acetic acid, Sigma) was added to a final concentration of $1 \mathrm{mM}$ in order to induce degradation of Rim4. For pdr5 mutant strain, proteasome inhibitor MG-132 was added at $6 \mathrm{~h}$ to $50 \mu \mathrm{M}$. For NDT80-IN (Benjamin et al., 2003) strains, at $\mathrm{t}=6 \mathrm{~h}, \beta$-estradiol was added to a final concentration of $1 \mu \mathrm{M}(5 \mathrm{mM}$ stock in ethanol, Sigma E2758-1G) to induce expression of NDT80 from the GAL1-10 promoter by activating the Gal4.ER fusion protein. For sporulation of wild strains, the procedure was essentially the same with the 


\section{Developmental Cell}

OPEN ACCESS

exception that if tetrads were already observed after overnight on YPG plates, they were pulled again from - $80^{\circ} \mathrm{C}$ directly on YPD $4 \%$, and the protocol was continued from there. Time points were taken for Western and Northern blot analysis and immunofluorescence/ DAPI as indicated.

For mitotic Ndt80/Sum1 experiments, strains were grown overnight in YPG (glucose in YPD would inhibit the GAL1-10 promoter driving NDT80) and diluted to $\mathrm{OD}_{600}=0.3$. Following transfer to fresh YPG medium, cells were incubated with vigorous shaking for $3 \mathrm{~h}$. Then auxin (indole-3-acetic acid, Sigma) was added $(\mathrm{t}=\mathrm{Oh}$ ) to a final concentration of $1 \mathrm{mM}$ in order to induce degradation of Sum1. After 1 hour, $\beta$-estradiol was added to a final concentration of $1 \mu \mathrm{M}(5 \mathrm{mM}$ stock in ethanol, Sigma E2758-1G) to induce expression of NDT80 from the GAL1-10 promoter by activating the Gal4.ER fusion protein. Time points were taken for Western and Northern blot analysis as indicated (collected volumes were adjusted to the $\mathrm{OD}_{600}$ at each time point).

For pheromone exposure experiments, strains were grown overnight in YPD and diluted to $\mathrm{OD}_{600}=0.2$. Following transfer to fresh YPD medium, cells were incubated with vigorous shaking for $3 \mathrm{~h}$. Cells were pelleted and resuspended at an $O D_{600}$ of 0.36 ( $t=0 \mathrm{~h}$ ), then $\alpha$-factor (Genscript) was added to a final concentration of $0.8 \mu \mathrm{g} / \mathrm{mL}(0.5 \mu \mathrm{M}$, "+") or $16 \mu \mathrm{g} / \mathrm{mL}$ (10 $\mu \mathrm{M}$, "++") in order to induce Ty3 activation. Time points were taken for Western and Northern blot analysis as indicated (collected volumes were adjusted to the $\mathrm{OD}_{600}$ at each time point).

\section{Meiotic Progression Analysis}

Cells were fixed in $3.7 \%$ formaldehyde. For analysis of nuclear divisions and spindle visualization, indirect immunofluorescence was performed as in (Carpenter et al., 2018) with minor modifications. To visualize spindles, we used rat anti-tubulin antibody (Bio-Rad) at a dilution of 1:200, and anti-rat-FITC antibody (Invitrogen) at a dilution of 1:200. Immunofluorescence samples were mounted in ProlongGold (Invitrogen) that included DAPI. Acquisition of images was conducted using a DeltaVision microscope (GE Healthcare). Spindle morphologies ( $n=100$ cells per time point) were classified as in (Lee and Amon, 2001). Briefly, Metaphase I cells were defined as cells with a single DAPI mass spanned by a short, thick, bipolar, meiotic spindle. Anaphase I cells were defined as cells with two distinct (though not always separated) DAPI masses, and a single long spindle that spans both DAPI masses. Metaphase II cells were defined as cells with two separate DAPI masses with each spanned by a bipolar, short, thick, meiotic spindle. Anaphase II cells were defined as cells with four distinct (though not always separated) DAPI masses with two long spindles. Then, these four categories of cells were grouped under the label "cells in meiotic divisions".

For analysis of nuclear divisions only, cells were washed once with $100 \mathrm{mM}$ phosphate, $1.2 \mathrm{M}$ sorbitol buffer [pH 7.5], permeabilized with $1 \%$ Triton X-100 and stained with $0.05 \mu \mathrm{g} / \mathrm{ml}$ DAPI.

\section{Northern Blot Analysis}

Samples were prepared by snap freezing the pellet of $7.2 \mathrm{OD}_{600}$ units of cells, and total RNA was isolated using a (400 $\left.\mu \mathrm{L}: 400 \mu \mathrm{L}\right)$ mixture of TES buffer (10 mM Tris [pH7.5], $10 \mathrm{mM}$ EDTA, 0.5\% SDS) and acid phenol: chloroform 5: 1 (Ambion) with zirconia/silica beads (BioSpec) while shaking at $1400 \mathrm{rpm}$ (Thermomixer, Eppendorf) at $65^{\circ} \mathrm{C}$ for $30 \mathrm{~min}$. After centrifugation at $16,000 \mathrm{~g}$ for $5 \mathrm{~min}$, the supernatant was ethanol precipitated at $-20^{\circ} \mathrm{C}$ overnight $(1 \mathrm{ml} 100 \%$ ethanol and $40 \mu \mathrm{l} 3 \mathrm{M}$ sodium acetate [pH 5.5]), centrifugated at $16,000 \mathrm{~g}$ for $20 \mathrm{~min}$, washed in $80 \%$ ethanol, dried, and resuspended in DEPC-treated water. For wild strains, RNA extraction required two chloroform purification. $5 \mu \mathrm{g}$ of RNA was denatured at $55^{\circ} \mathrm{C}$ for $15 \mathrm{~min}$ in $50 \%$ formamide, $18.3 \%$ formaldehyde, and $5 \%$ MOPS and was resolved on a denaturing agarose gel (1.9\% agarose, $3.7 \%$ formaldehyde, $1 \times$ MOPS buffer) for 2.5 hours at $80 \mathrm{~V}$. RNA was transferred to a Hybond-N+ membrane (GE Healthcare) by capillary transfer in 10x SSC (1.5 M NaCl, $0.15 \mathrm{M}$ trisodium citrate dihydrate, [pH 7]). The membrane was stained with methylene blue, incubated in hybridization Buffer (0.25 M Na-Phosphate [pH 7.2], $0.25 \mathrm{M} \mathrm{NaCl}, 1 \mathrm{mM}$ EDTA, $7 \%$ SDS, and $5 \%$ dextran sulfate) at $65^{\circ} \mathrm{C}$ and probed with ${ }^{32} \mathrm{P}-$ labeled PCR products prepared via Amersham Megaprime DNA labeling kit (GE Healthcare) and Illustra ProbeQuant columns (GE Healthcare), transferred to a phosphor screen, and imaged on a Typhoon Trio variable mode imager (Amersham).

To observe Ty3 dimers we conducted semi-denaturing Northern blot analysis (adapted from (Nymark-McMahon et al., 2002)). Briefly, the procedure is as above except we omitted formamide in the denaturing mix and incubated 15 minutes at $30^{\circ} \mathrm{C}$.

\section{Yeast RNA Sequencing and Sequencing Analysis}

Briefly, poly-A pull-down was used to enrich mRNAs from total RNA samples and proceed on library preparation by using Illumina TruSeq RNA prep kit. Libraries were sequenced using single-end sequencing (100 bp) in multiplex using Illumina HiSeq4000 at Columbia Genome Center. RTA (Illumina) was used for base calling and bcl2fastq2 (version 2.17) for converting BCL to fastq format, coupled with adaptor trimming. Sequencing reads were mapped onto SK1 genome reference (Yue et al., 2017) using STAR (Dobin et al., 2013) with the following parameters: Multiple alignments allowed: 500 (takes into account the number solo-LTR loci in the genome); Multiple alignment retained: 1; Order of multiple alignments: Random (-outFilterMultimapNmax 500 -outSAMmultNmax 1 -outMultimapperOrder Random -outFilterScoreMinOverLread 0.3 -outFilterMatchNminOverLread 0.1 -seedSearchStartLmax 20 -seedSearchStartLmaxOverLread 0.2) while other parameters were set to defaults. Reads count were scaled by CPM (count per millions) and visualized using Ving software (Descrimes et al., 2015).

\section{Immunoblot Analysis}

The pellet of $7.2 \mathrm{OD}_{600}$ units of cells was resuspended in $5 \% \mathrm{TCA}$, incubated overnight at $4^{\circ} \mathrm{C}$, washed with acetone, and dried. Cells were broken using $50 \mu$ lacid-washed glass beads (Sigma), $100 \mu$ lysis Buffer (10 mM Tris-HCl [pH 11], 1 mM EDTA [pH 8], 2.75 mM 
DTT, Halt protease inhibitors (Thermo Fisher)), and a $45 \mathrm{sec}$ process in a FastPrep-24 (MP Biomedicals) at max speed. $50 \mu \mathrm{L}$ Loading Buffer (9\% SDS, $0.75 \mathrm{mM}$ Bromophenol blue, $187.5 \mathrm{mM}$ Tris- $\mathrm{HCl}$ [pH 6.8], 30\% glycerol, and 810 mM $\beta$-mercaptoethanol) was added, samples were heated at $100^{\circ} \mathrm{C}$ for 5 mins, and centrifuged 5 mins at 20,000 g. Polyacrylamide gels (precast $10 \%$ Criterion TGX Precast Midi Protein Gel 26 wells, Bio-Rad) were run on a midi gel system (Criterion Vertical Electrophoresis Cell, Bio-Rad) with SDS Running Buffer (190 mM glycine, $25 \mathrm{mM}$ Trizma base, $3.5 \mathrm{mM}, 1 \%$ SDS), with $4 \mu \mathrm{l}$ sample per well. They were transferred using a semi-dry transfer apparatus (Trans-Blot Turbo Transfer System, Bio-Rad) to a nitrocellulose membrane (Trans-blot turbo transfer kits Nitrocellulose, Bio-Rad), and stained in Ponceau.

$\alpha$-VLP antibody was purified from $\alpha$-VLP (Ty3) crude serum (gift from Sandmeyer lab), using Gag3 protein produced from pLZL2422 (Larsen et al., 2008) coupled to an AminoLink column (Thermo).

Antibodies were prepared in 1X TBST with 1\% milk 1\% BSA. Primary antibodies were used at the following concentrations: $\alpha$-VLP (purified from $\alpha$-VLP (Ty3) crude serum, gift from Sandmeyer lab) 0.1ug/mL, $\alpha$-HA.111:1,000 (BioLegend), $\alpha$-V5 1:2,000 (Invitrogen), $\alpha$-FLAG (Sigma) 1: 800, $\alpha$-Pgk1 1: 20,000 (Novex). $\alpha$-mouse or $\alpha$-rabbit HRP-conjugated secondary antibodies (GE Healthcare) were used.

\section{Ndt80 ChIP-seq}

NDT80-3V5 and no tag control cells were sporulated at $30^{\circ} \mathrm{C}$. Cells were fixed in $1 \%$ formaldehyde for 15 minutes at room temperature and quenched with $0.1 \mathrm{M}$ glycine (final concentration). Samples were prepared by snap freezing the pellet of $20 \mathrm{OD}_{600}$ units of cells. Cells were resuspended in $0.4 \mathrm{ml}$ lysis buffer $(50 \mathrm{mM} \mathrm{HEPES} / \mathrm{KOH} \mathrm{pH}$ [7.5], $140 \mathrm{mM} \mathrm{NaCl}, 1 \mathrm{mM}$ EDTA, $1 \%$ Triton-X, $0.1 \% \mathrm{Na}-$ deoxycholate, $1 \mathrm{mM}$ PMSF) lysed with zirconia/silica beads (BioSpec) in a FastPrep homogenizer. The homogenate was centrifuged at 2,500 rpm for 1 minute and the supernatant was discarded. The pellet was resuspended in $0.25 \mathrm{ml}$ lysis buffer and sonicated (15 sec on, $30 \mathrm{sec}$ off, 10 cycles). Lysate was centrifuged at $10,000 \mathrm{~g}$ for 5 minutes at $4^{\circ} \mathrm{C}$ and supernatant was transferred to a new tube. $50 \mu \mathrm{L}$ was taken for DNA input sample and the remaining sample was processed for ChIP.

Ndt80-DNA complexes were immunopurified using anti V5 agarose beads (Sigma) for $1 \mathrm{~h}$ at $4^{\circ} \mathrm{C}$. Beads were washed and crosslinks were reversed using Chelex resin (Bio-Rad). Elution was carried out by boiling samples for 10 minutes followed by Proteinase $\mathrm{K}$ digestion $\left(1 \mathrm{mg} / \mathrm{ml} 30 \mathrm{~min}\right.$ at $\left.55^{\circ} \mathrm{C}\right)$. Beads were boiled again for 10 minutes and the eluate was collected and purified/concentrated using a Qiagene Minelute kit. Sequencing libraries were prepared from the eluate using an Accel-NGS 1S plus (Swift Biosciences) per the manufacturer's protocol. Libraries were sequenced using paired-end sequencing (2x100 bp) in multiplex using NextSeq 550 .

\section{QUANTIFICATION AND STATISTICAL ANALYSIS}

Immunoblot and Northern blot were quantified using FIJI software (Schindelin et al., 2012). Signal intensity was normalized to a loading control (Pgk1 for immunoblots, 25S rRNA for Northern blots). Values were multiplied by an arbitrary scaling factor, which was held constant within each figure panel for each technique. Statistical significance was determined by Student's T-test or Ztest statistic as indicated. Number of biological replicates is indicated in figure legends where applicable.

Statistical Analysis for Ndt80 Binding Site Enrichment Upstream Ty3 Insertions

Data analysis was performed using the R project for statistical computing version 3.6.1 (https://www.r-project.org) and RStudio version 1.1.383 (https://www.rstudio.com), utilizing the following packages: dplyr, tidyr, stringr, ggplot2, plotly, tools, boot, Biostrings, GenomicRanges, janitor, seqinr, BiocManager, TFBSTools. Briefly, yeast genomes were downloaded from (Yue et al., 2017) and Ndt80 binding sites (defined as CACAAA by (Nocedal et al., 2017)) were identified. Then the positions of Ndt80 binding site upstream Ty3 elements (fulllength or solo-LTRs) were recorded. For Ndt80 target genes and non-target genes in SK1, the same process was applied, using the SK1 reference genome and annotation from (Nocedal et al., 2017). For bootstrap, the R function sample_n was used to take samples with replacements. Then the data was visualized using $50 \mathrm{bp}$ long non-overlapping bins. We used the distribution of outlier boundaries for each bootstrap experiment to identify region of significant difference in Ndt80 binding site enrichment.

\section{Analysis of Yeast ChIPseq Data}

Reads were trimmed of their adaptors using cutadapt (Martin, 2011) and were aligned to the S. cerevisiae SK1 genome (Yue et al., 2017) using Bowtie2 version 2.3.4 (Langmead and Salzberg, 2012). Alignments were filtered using samtools v1.6 (Li et al., 2009) (samtools view -f 2 -F 1804 -q 30), and PCR duplicates were filtered out using Picard tool MarkDuplicates v2.23.3 (Broad Institute). Deeptools bamcoverage (Ramírez et al., 2016) was used to generate a coverage track of number of reads per bp after reads were extended to their mates, to reflect the actual fragment length. Coverage was then normalized using a script detailed in (De Muyt et al., 2018): upon the assumptions that for a transcription factor, peaks are expected to be narrow, they are expected to fall in the top outliers of the coverage distribution, while the background regions are expected to fall in the bulk of the distribution, between Q1-1.5IQR and Q3+1.5IQR. Hence, for untagged immunoprecipitate (UIP), the coverage was divided by the average read count in non-null and non-outlier positions, while for tagged immunoprecipitate (IP), the coverage was divided by the average read count in non-null and non-outlier positions and multiplied by the average read count of the same set of positions in the normalized UIP. Normalized profiles were used for peak detection using bpeaks v1.2 (Merhej et al., 2014) using as thresholds: T1: IP>(Q3 $\log _{2} \mathrm{FC}>0.5$, and T4: $\left(\left(\log _{2}(\mathrm{IP})+1\right)+\left(\log _{2}(\right.\right.$ control $\left.\left.)+1\right)\right) / 2>0$. For visualization on IGV (Thorvaldsdóttir et al., 2013), normalized UIP profiles were subtracted to their corresponding normalized IP profiles using Deeptools bigwigCompare (Ramírez et al., 2016). 
Analysis of Mammalian RNAseq Data (Mouse, Opossum)

RNAseq from enriched populations of adult mouse testicular cell types (Soumillon et al., 2013) was downloaded from EBI ENA:PRJNA187158 (https://www.ebi.ac.uk/ena). Reads were trimmed to remove adapter sequences (stringency 5 -length 20) using TrimGalore 0.4.1 (https://github.com/FelixKrueger/TrimGalore) then aligned to the mm10 reference assembly of the mouse genome with Tophat v2.1.0 (Kim et al., 2013) (command line options -g 1 -no-coverage-search -b2-sensitive) using the GencodeBasic VM18 transcriptome as a transcriptome index (Frankish et al., 2019). Accepted hits from Tophat were filtered with samtools v1.6 (Li et al., 2009) to remove unmapped reads and non-primary alignments. For locus-specific analysis, uniquely mapping reads were selected by removing reads with a quality score below 5 . Reads overlapping Repeatmasker-annotated loci in the mouse genome were counted for each repeat locus, and aggregated at the level of the repeat name (repName). EdgeR (Robinson et al., 2010) was then used to normalize the counts per million mapped fragments in each sample using trimmed means. CPMs were converted to fragments per kilobase of transcript per million mapped reads (FPKM) by dividing CPM by the length of the repeat locus for locus-specific analysis, or by the mean length of repeat loci annotated with that repeat name for repName-level analysis.

RNAseq for P14 Myb/1 testes (Zhou et al., 2017) and Stra8 preleptotene spermatocytes (Kojima et al., 2019) were downloaded from EBI ENA:PRJNA476515, PRJNA476515, trimmed and mapped to mm10 as described above. The paired end Myb/1 data was mapped using mate inner distance and mate standard deviations of 30 and 68 respectively. Accepted hits from Tophat were filtered as described above and uniquely mapping reads falling within GencodeBasic VM18 genes counted using htseq-count v0.11.2 (Anders et al., 2015) in intersection-nonempty mode. The ENSMUST00000174651.1 transcript in the GencodeBasic VM18 annotation, which appears to be a Moap1 transcript originating from an alternative upstream promoter, was manually re-assigned from the RP24234J3.3 gene to Moap1 to circumvent ambiguity in htseq-count assigning reads to Moap1. EdgeR was used to normalize the counts per million mapped fragments in each sample using trimmed means, data for genes of interest (Moap1, Pnma1) selected from the dataset, and Student's t-test used to assess statistical significance between $\mathrm{Myb/1^{+/- }}$ and $\mathrm{Myb} / 1^{-/-} \mathrm{P} 14$ testis samples, or between $\mathrm{Stra8}^{+/-}$highSTRA8 preleptotene spermatocyte and Stra8 ${ }^{-/-}$preleptotene spermatocyte samples.

FPKMs from RNA-seq for opossum from purified spermatocyte/spermatid and multitissue (Lesch et al., 2016; Marin et al., 2017) were downloaded from NCBI GEO: GSE68507, GSE97367.

\section{Analysis of Mammalian ChIPseq Data (Mouse)}

Mybl1 and Stra8 ChIPseq data from mouse testes (Kojima et al., 2019; Zhou et al., 2017) were downloaded from EBI ENA:PRJNA321732, PRJNA476515, trimmed as described for RNAseq data, then aligned to the mm10 assembly of the mouse genome using Bowtie v2.2.6 (Langmead and Salzberg, 2012). Bowtie output was filtered to remove PCR duplicates marked by PicardTools v2.17.11 (http://broadinstitute.github.io/picard/), and to remove reads mapping to mitochondrial or blacklisted regions of the genome (https://www.encodeproject.org/files/ENCFF023CZC/), unmapped reads, non-primary alignments and reads with a quality score below 5. Bedtools (Quinlan and Hall, 2010) was used to convert the Bowtie alignments to ENCODE tagAlign format, and MACS v2.1.1 (Zhang et al., 2008) used to call peaks on pooled ChIP samples relative to pooled input controls using default settings $(q<0.05)$ and to generate Bedgraphs showing fold enrichment in pooled ChIP relative to input samples. For Stra8 ChIPseq, the fold-enrichment for anti-FLAG ChIP in control wild-type samples was then subtracted from the fold-enrichment for the anti-FLAG ChIP in experimental Stra8 ${ }^{F L A G / F L A G}$ samples. For Mybl1 ChIPseq, anti-Mybl1 ChIPseq from Myb/1 ${ }^{+/-}$testes was compared to $\mathrm{Mybl1}^{+/-}$input controls. ChIPseq bedgraphs and peak co-ordinates were visualised using Gviz (Hahne and Ivanek, 2016). Distances from Repeatmasker-annotated MamGyp-int loci and GencodeBasic VM18 genes overlapping those loci to the nearest Stra8 and Mybl1 ChIPseq peaks were determined using BEDtools v2.26.0 (Quinlan and Hall, 2010).

Analysis of Dazl CLIP and DAZL RIP Mammalian Data (Mouse, Human)

Dazl CLIP tag locations from mouse testes (Zagore et al., 2018) were downloaded from GEO:GSE108181 and the genomic co-ordinates lifted over from mm9 to mm10 using the UCSC liftOver tool (Haeussler et al., 2019). DAZL RIP data from human ovaries (Rosario et al., 2017) (available in EBI ENA:PRJNA321858) was trimmed and aligned to the hg38 assembly of the human genome as described for the RNAseq data using a mate inner distance of 260 and mate standard deviation of 110 . Tophat output was filtered, then reads in genes or repeats counted using htseq-count or BEDTools and normalized in EdgeR using the total number of mapped fragments as the library size as described for the RNAseq data. To assess MOAP1 and PNMA1 behaviour in the htseq-count data, EdgeR, low abundance genes were removed using filterByExpr(count=15, min.count=10), gImLRT used to determine differentially abundant genes in a paired experimental design, MOAP1 and PNMA1 data extracted from the resulting DGELRT object. and false discovery rate used to correct $p$-values for multiple-testing. To determine enriched retrotransposon sequences in the anti-DAZL samples, repName-aggregated repeat count data for retrotransposons (LINE, SINE and LTR repeat classes) were selected then low abundance retrotransposons removed using filterByExpr(count=15, min.count=10). glmLRT was used to determine differentially abundant retrotransposons in a paired experimental design, and false discovery rate used to correct $p$-values for multiple-testing. Data for retrotransposons belonging to the Gypsy repeat family were plotted. 